\title{
Article
}

\section{Collaborative Workplace Design: a Knowledge-Based Approach to Promote Human-Robot Integration and Multi-Objective Layout Optimization}

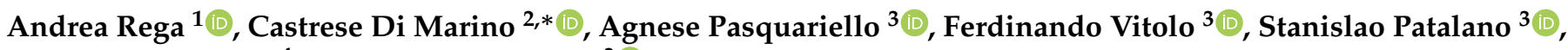 \\ Alessandro Zanella ${ }^{4}$ and Antonio Lanzotti ${ }^{3}$ (D) \\ 1 Department of Neurosciences, Reproductive and Odontostomatological Sciences, University of Naples \\ Federico II; andrea.rega@unina.it \\ 2 Department of Management, Information and Production Engineering, University of Bergamo; \\ castrese.dimarino@unibg.it \\ 3 Fraunhofer J-Lab IDEAS, Department of Industrial Engineering, University of Naples Federico II; \\ ferdinando.vitolo@unina.it; agnese.pasquariello@unina.it; stanislao.patalano@unina.it \\ 4 Centro Ricerche FIAT SCpA, Orbassano, Italy; alessandro.zanella@crf.it \\ * Correspondence: castrese.dimarino@unibg.it
}

\begin{abstract}
The innovation driven Industry 5.0, in agreement with Industry 4.0, leads to consider human in a prominence position as the center of manufacturing field. This pushes towards the hybridization of manufacturing plants promoting a fully collaboration between human and robot. Furthermore, the new paradigm of "human centred design" and "anthropocentric design" allows enabling a synergistic combination of human and robot skills. However, properly collaborative workplaces are currently very few. Industry is still not confident, and systems integrators hesitate to venture into Human-Robot Collaboration (HRC). Despite the effort in collaborative robotics, a general solution to overcome the current limitations in designing of collaborative workplaces still misses. In the current work, a Knowledge-Based Approach (KBA) is adopted to face collaborative workplace designing problem. The framework resulting from the KBA allows developing a modelling paradigm that enable to define a streamlined approach for the layout designing of a collaborative workplace. Finally, a what-if analysis and a ANOVA analysis are performed to generate and evaluate a set of scenarios related to a collaborative workplace for quality inspection of welded parts. Facing the high complexity and multidisciplinary of HRC can be conveyed to develop a general design approach aimed at overcoming the difficulties that limit the spread of HRC in the manufacturing field.
\end{abstract}

Keywords: Collaborative robotics; Human-Robot Collaboration (HRC); Knowledge-Based Approach (KBA); collaborative workplace design; systematic layout planning; digital layout optimization; what-if analysis.

\section{Introduction}

Nowadays, the innovative paradigm of "Industry 5.0" complements the existing "Industry 4.0" approach by counting on a human-centric industry. The hybridization of manufacturing plants with the collaboration between human and robot was one of the main pillars of the "Industry 4.0" framework and it becomes stronger within the "Industry 5.0" framework [1,2]. Furthermore, robotics is assuming an increasingly central role in manufacturing field due to innovation in automation and because of the market pressure. The market and the companies acknowledge in robotics and collaborative robotics the future of manufacturing and adopt willingly the innovation carried on thus far by the industry 4.0 and still today by the industry 5.0. Collaborative robotics offers the opportunity for replacing human in all the tasks and operations supposed alienating, bringing out his new role inside the collaborative workplace. 
Even thought a high level of automation allows reducing the cost production [3], it reduces the production flexibility which is the main characteristic of human-based workplace. Therefore, in the production context of mass customisation, human takes on key role in the flexible manufacturing field. The new paradigm of "human centred design" and "anthropocentric design" [4,5] is approaching the plant and workplace design by enabling a synergistic cooperation of human skills and new industrial equipment. Therefore, despite the significant strength of the automation, there are still several applications where human presence is essential. In such a context, Human-Robot Collaboration (HRC) - performing common tasks by sharing the same workspace - is elevated to an enabling solution for mass customisation which needs a high degree of flexibility [6]. Moreover, it is essential whenever either the processes are hard to be automated or affected by uncomfortable operations. However, properly collaborative workplaces are currently very few. Most applications involve fenced cobots used as traditional industrial robots, or workplaces where the human and the cobot coexist and perform different tasks on different workpieces, without sharing tasks and/or space. A maze of standards, rules and guidelines [7], and the lack of systematic approach to HRC workplace designing are the main obstacles to a properly and efficient collaboration. Too often there is a conflict between unnecessary safety measures and productive needs. For these reasons, industry is still not confident and system integrators are hesitant to venture into HRC.

The current paper proposes a Knowledge-Based Approach (KBA) to face with the mess and uncertainty characteristics of such complex scenarios. Thus, the KBA aims at encouraging the implementation of collaborative workplaces and the exploitation of the full potential of HRC. The collection, study, organization and exploitation of the knowledge about HRC allowed creating a strong basis framework. This rigorously identifies all the elements of the collaborative workplace and defines relationships among them. By means of this framework, the designer can outline the changes and their influence on all identified elements. Consequently, all this is used to develop a modelling paradigm that clusters the identified elements and the critical features that affect the workplace layout definition. Via the KBA and the paradigm, it is possible to understand and visualize how all the elements interact each others and which output a certain configuration of them can produce. Based on these considerations, it is proposed a designing approach composed of four main steps for optimal positioning of the resources by using ad-hoc alghoritms. Finally, a what-if analysis and a ANOVA analysis are performed by the generation and evaluation of a set of scenarios related to an industrial use case.

\subsection{Structure of the work}

This work deals with the collaborative robotics issue and presents an innovative designing approach for collaborative workplaces. In the section 2, a detailed state of art about collaborative robotics is reported and discussed. The main aspects and problems connected to collaborative robotics are faced and deepen.

In the section 3, a Knowledge-Based Approach (KBA) is adopted to take care of collaborative workplace design. The KBA is composed of three phases:

1. The Knowledge Acquisition dealing with the exploration of HRC field.

2. The Knowledge Management dealing with the organization of the collected information.

3. The Knowledge Representation dealing with the representation of the designing output.

The acquisition is carried out by collecting the raw data and information connected to the HRC. This knowledge converges into five dominions of the collaborative robotics. The management tackles the organization of the information as well as the definition of the relationship among all the elements in a collaborative workplace. The representation 
is done by means of the visualization and manipulation of the information in order to make it available for the designer.

The design of the collaborative workplace layout is presented in section 4 . In section 4.1 the modelling paradigm is explained. Consequently, the problem formalization and the proposed approach are detailed. To achieve the implementation of the method, all the organized knowledge is traduced in the form of an objective function. The objective function takes care of the knowledge about HRC and points to the minimization of the floor space respecting the minimum distances among the resources and the ergonomic working conditions. Afterwords, the whole framework can be visualized by means of $3 \mathrm{D}$ visual environment.

The application of the proposed approach is presented in section 5. A use case about a collaborative quality inspection is introduced in section 5.1. Consequently, a what-if analysis is presented in section 5.2. A set of 27 configurations of the workplace is generated through the combination of three different parameters (minimum distance among the resources, relative position of the logistic areas and robot speed), changing on three different levels. In section 6, the results of the study are presented and explained by means of the Simple Multi-Attribute Rating Technique (SMART) and the ANOVA analysis. Finally, in section 7, the conclusions of the work are summarized.

\section{State of art}

The main purpose of this paper concerns the designing and optimization of the collaborative workplace layout. The facility layout problem is a well-known and widely addressed topic in the last decades [8-10]. However, in the context of collaborative robotics, it becomes a very complex problem to deal with, due to the several strongly coupled issues. Thus, the most recent works addressing the topics intrinsically linked to the design and optimization of the collaborative workplace are reported below.

Scimmi et al. [11], for example, use an assembly cell layout prototype to investigate the benefits coming from the human-robot collaboration. Their work is focused on the robot control strategy within the proposed layout. It proposes a collision avoidance algorithm based on repulsive velocities. The main focus of this work is related to robot control and programming, a technical problem clearly related to the design of an efficient layout.

Following the same selection criterion, the State of Art (SoA) collects a series of works that propose tools and methods to tackle task allocation problem, ergonomics aspects, interaction between the human being and the machines, safety and standards compliance, management problems.

Task allocation is one of the most largely addressed topics in this context. Several works proposed methods, approaches and tools which aim to fully exploit the potential of both robots and humans starting from an optimized task allocation. Murali et al. [12] propose an architecture based on AND/OR graphs for representing and reasoning upon human robot collaboration in real-time. The aim of the work is to develop task planning algorithms that can recognize and adapt to human action. In this way, a real and more efficient collaboration between human being and robot could be reached. Mateus et al. [13] develop an algorithm for identifying the task precedence in an assembly operation. The aim of this paper is to overcome the rigid linear assembly sequences and enable a more flexible collaborative assembly. Banziger et al. [14] propose a method to allocate tasks to human and robot in an optimized way for a given workplace. The method leverages a standardized work description. The authors propose an approach to optimize the human-robot work organization by means of genetic algorithms. The authors demonstrated that, using the proposed method, they can optimize the work organization in terms of production time and ergonomics for an existing workplace.

El Makrini I. et al [15] propose a framework for task allocation in human-robot collaborative application based on the agents' capabilities and on ergonomics considerations. Suitable agents are defined considering their characteristics, task requirements 
and workpieces features; ergonomics considerations are made by measuring the posture of the human body and the related workload.

Ergonomics is another key topic in the field of collaborative robotics. Collaborative robotics can be seen as a means of improving the working conditions of the human operator and, at the same time, making the production process more efficient $[16,17]$. However, it is necessary to consider physical and mental wellness of the human since the coexistence with a robot can make less tiring some task but, at the same time, it could produce stress for the operator [18]. Both physical and cognitive ergonomics need to be assessed. The physical ergonomics is related to posture, lifting and transport, repeated movements and involves the musculoskeletal disorders [19]. It can be evaluated by means of the following well-known methodologies: (i) the National Institute for Occupational Safety and Health (NIOSH) [20] equations for manual lifting of objects; (ii) the Rapid Upper Limb Assessment (RULA) [21] for postures; (iii) the Occupational Repetitive Action (OCRA) [22] for repetitive tasks. Cognitive ergonomics is mostly linked to factors not objectively measurable and connected to the mental wellness and represents an aspect that should not be neglected when a human works next to a robot [23]. Indeed, the mental workload is the ability to make decisions and to face stress.

Elias Matsas et al. [24] investigate the acceptability of human robot collaboration in terms of mental safety issues, i.e., human's awareness and vigilance of the moving robot. They present a highly and immersive Virtual Reality Training System (VRTS) named "beware of the robot" that simulates in real-time the cooperation between industrial robotic manipulators and humans, executing simple manufacturing tasks.

Considering the interaction between human and robot, on the one hand the reference standards [25-27] suggest safety-based interaction between human and robot, on the other hand several researchers propose different degrees of interaction no longer based only on the safety functions but considering the way they interact and carry out the tasks [5,28]. Ali Ahmad Mailk and Arne Bilberg [29] describe the interaction between human and robot by means of an architecture based on three-dimensional reference scale: team composition, level of engagement and safety are the three considered dimensions. Finally, [30-32] define different levels of interaction.

In [33], the authors propose the Digital Twin (DT) approach to address the complexity of achieving the full potential of human-robot collaboration in assembly application. It proposes the idea of digital twin as a "front runner". In this way it is possible to optimize the behaviour of the physical system creating its time dependent accurate virtual model and simulating it. This approach takes into account each change introduced and could predict the future behaviour of the system without financial loss or human injury.

Kousi et al. [34] proposed a DT based approach for designing and redesigning flexible assembly system. The work proposes as use case a scenario in which humans and an autonomous mobile dual arm collaborate. The advantage resulting from the use of the DT is the possibility to dynamically update or reconfigure the scenarios in response to the occurrence of unexpected events.

However, the DT has the disadvantage of requiring a real scene and relatively expensive equipment to fully exploit the potential use.

In other words, the DT has to be associated with an object that actually exist. "A digital twin without a physical twin is a model" [35]. This aspect, in some circumstances, e.g., early design of a workplace, could be seen as an impediment. "The DT for design is only meaningful when the prototyping stage is reached" [35].

Boschetti et al. [36] focus on the aspects related to the process and on the evaluation of its performance. The work proposes a model in order to estimate the cycle time of collaborative assembly system using information about process as input. The paper presents a mathematical model useful to evaluate the feasibility of a multi-resource collaborative assembly system and for making the most appropriate choices in the design phases. 
In [37], the authors investigate the influence of the product characteristics on Human-robot collaboration. The authors developed an algorithm which simulates the product assembly in order to estimate the makespan realizable for several scenarios.

Vitolo et al. [38] propose a multi-layer modelling approach to systematically identify potential collaborative workstations within an industrial production plant, providing a tool to solve another challenging topic in the HRC context.

$[39,40]$ present different methods to assess the potential benefits of a conversion from a manual workplace into a collaborative one.

In [41], Tsarouchi et al. pursue a goal that may be considered more close to that of the present manuscript. They face the problem of HRC workplace design starting from the task planning. Alternative layouts and task allocations are obtained by means of a multi-criteria decision making framework. Then the different alternatives were evaluated. The approach does not consider the compliance with the reference standard in the decision making framework.

Ore et al. [42] identified the lack of methods supporting efficient HRC workstation design. They propose a step-by-step method useful in the early phases of the workstation design in the context of the Human-Industrial Robot Collaboration (HIRC). The method is based on the Pahl and Beitz's engineering design framework. It was used to design an assembly cell in the automotive context. The work highlighted that advanced virtual simulation software should be used to generate more accurate data into the decision process.

Lietaert et al. [43] present a methodology to optimize the design of a collaborative workplace according to spatial and ergonomic criteria. The authors taken into account a detailed ergonomic evaluation in the formalisation of the optimization problem, but do not consider the standard constraints. The proposed approach was evaluated in a sheet metal manufacturing case study.

Mateus et al.[44] proposed a structured designing method addressing fundamental aspects for the designing of collaborative workplaces. They focused in particular on safety, ergonomics, time and performance.

Although [41-44] look in the same direction as the current work, they do not place enough emphasis on the safety aspect.

In [45], the authors focus their work on the safety for human operator. They propose an industrial robot performs which a depalletizing operation as use case. The robot acts in a speed and separation monitoring mode, and human operators move in the surrounding space. There are no physical fence and a separation distance between human and robot must be guaranteed according to the ISO TS 15066 . Usually the separation distance is calculated considering the worst-case. The paper proposes an alternative approach useful to reduce the area of the workplace layout dedicate to the separation distance. However, the work is focused on the operations performed in the speed and separation monitoring mode.

Valid solutions and tools have been proposed but they solve only partial problems. The proposed tools and solutions, as well as the standards, are not enough for the successful development of a such complex system. The actual implementation of collaborative workplaces is hindered by the lack of an overall view of the topic. An approach which considers all the issues related to collaborative robotics and especially their relationships is still missing. It is necessary an appropriate methodology which can lead to an optimal exploitation of the proposed solutions or tools, by providing relationships, interactions and rules to consider during the design phase. Gervasi et al. try to face the identified lack providing an analysis on several aspect, from robotics to human factors. In [46] they propose eight latent dimensions in HRC context and develop a framework for HRC applications assessment.

The Knowledge-Based Approach presented in the next section has the same aim. The KBA allowed to breakdown the HRC domain in a hierarchical structure which is composed of elements and sub-elements most often linked by close relationship 
of interdependence. Finally, on the basis of the developed framework, an approach to HRC workplace layout design, which takes into account the issues highlighted by the academic research, the needs of the industry, the safety regulatory precepts was developed.

The proposed design approach allowed to reduce the "routine-like" and "non-value adding" design tasks which require little creativity. In this way, designers could make better use of their creativity and intuitiveness without being hindered by repetitive design tasks.

\section{A Knowledge-Based Approach for the investigation of collaborative workplaces}

The deepening of the of the Human-Robot Collaboration (HRC) that emerges from the study of the related State of Art, shows how it is a very complex problem to deal with, and brings attention on the need of a structured approach to face all the issues related to this topic. In this scenario, the only experience is not sufficient to fully understand and address the designing of a collaborative workplace. Accordingly, a Knowledge-Based Approach (KBA) that aims to face the complex problems related to collaborative robotics is proposed and implemented to achieve a general valid solution in this research field. Indeed, because of the amount and heterogeneity of the elements and information [47] involved in HRC, it is tough to go through all the related aspects without a structured framework. Due that, the KBAs are largely applied to many engineering fields [48] such as software architecture [49] or decision making support for the development of complex systems and structures [50]. For all these reasons, in this paper a KBA is adopted to face the high level of complexity about collaborative robotics [51].

The main purpose of the adopted KBA is to discover and organize the main information about collaborative robotics in order to make them available and exploitable to address collaborative workplaces. The approach involves to collect the information and knowledge related to the collaborative robotics and define the relations among all the elements that are part of collaborative workplaces. Consequently, all they are organized towards a structured framework, that is a supporting structure of methods and tools able to support the designing process. Finally, the application of the KBA leads to the development of a designing method to assist the designer in finding the best solution or configuration for specific situations. The KBA is composed of the three phases represented in Figure 1: (i) knowledge acquisition, (ii) knowledge management and (iii) knowledge representation.

\subsection{Knowledge acquisition}

The knowledge acquisition is the first part of the KBA and refers to the collection and study of documents and works about the collaborative robotics. The aim of this phase is to acquire the necessary knowledge, establish a set of relations and rules and

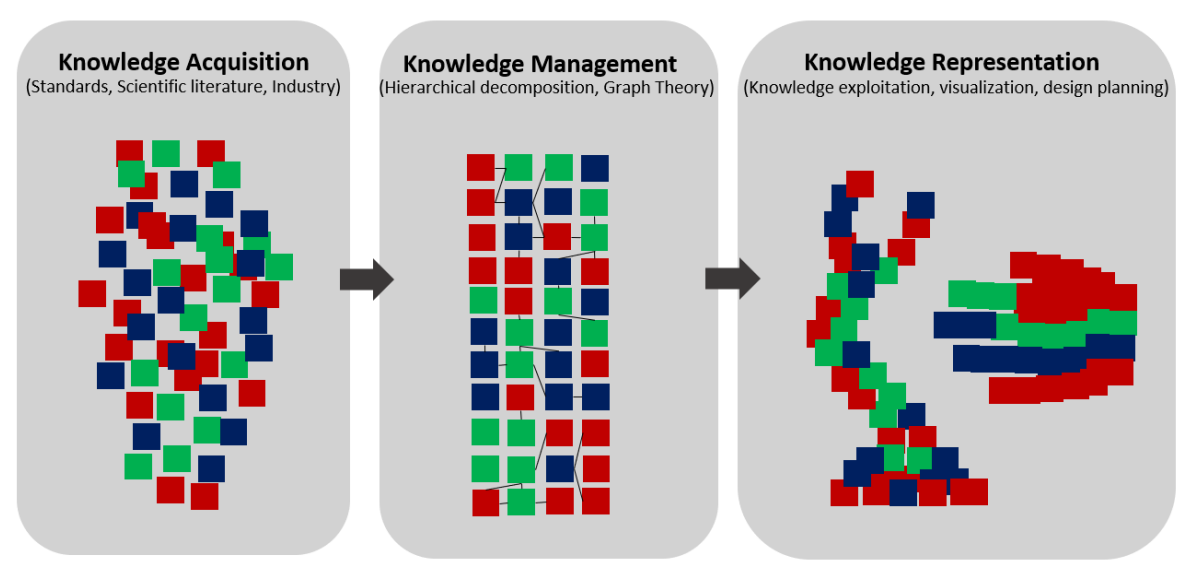

Figure 1. Depiction of the main phases of the Knowledge-Based Approach. 
generate usable datasets. The HRC field is analysed from three points of view: (i) the scientific literature, (ii) the regulatory framework and (iii) the industrial context. Besides, as described in section 2, the state of art about collaborative robotics is very extensive and includes a multitude of different topics. The overview provided in the previous chapter allows identifying the main areas of interest included in the present research work: task allocation, safety, ergonomics, human-robot interaction, facility layout problem, robot control and so forth. All these topics turned out to be interrelated and strictly coupled to the HRC workplace design issue.

In the same manner, the study and the critical analysis of the regulatory framework leads to the identification of the main aspects related to safety and risk reduction within a HRC workplace. However, a detailed discussion on standards is redundant considering that other works [52-54] already did it. It suffices to recall that the standards regarding robotics are organised into three types as follow:

- $\quad$ Type A standards: basic safety standards and requirements to apply to machinery.

- $\quad$ Type B standards: generic safety standards divided in two sub-categories.

- $\quad$ B1 standards concern specific safety aspects.

- $\quad$ B2 standards concern safeguard measures, interlocking devices, optical or pressure sensors.

- $\quad$ Type C standards: safety requirements for specific machinery.

Among the latter types, it is worth mentioning the ISO/TS 15066:2016 which is the most recent in collaborative robotics and concerns the interaction between human and robot presenting four collaborative operations. Furthermore, it is a technical specification which describes in detail the allowed collaborative operations and specifies their requirements. It reinforces the indications given in ISO 10218-1/2 (type C), that are the main standards about robots and robotic devices. For the purpose of the present work, the relations among the main standards involved in HRC are found starting from the three standards above mentioned. The connections are presented in the network displayed in Figure 2 where a link is the direct connections between two standards. The acronyms ISO and IEC include all the standards that are not directly involved in HRC but are preparatory for the adoption of these standards.

Still, an overview of collaborative robotics of the industrial context is given by [55] and [56]. The following key points arise:

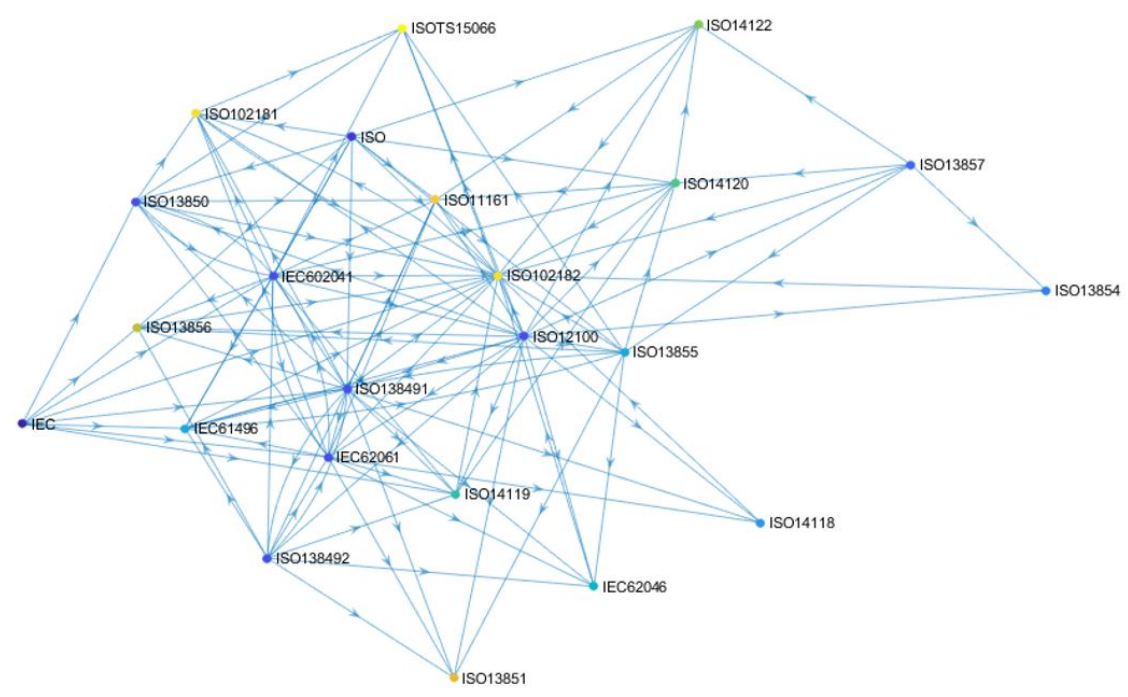

Figure 2. Network of relationship among the standards related to collaborative robotics 
- Grouping the applications of collaborative robots by sector, about three quarters of them belong to electrical engineering and automotive sectors. There are fewer applications in the plant engineering and mechanical fields.

- Focusing on the category of application, the majority of applications concern assembling or material handling.

- Applications where a worker really collaborates in strict contact with the cobot are not very common. In most of the cases, humans and robots coexist sharing the same workspace only occasionally. Furthermore, cobots are usually placed behind physical fence and used in the same way as classic industrial robots.

Considering the aspects presented on the above analysis, the knowledge about HRC needs to be captured and stored as rules, constraints, semantic representation and facts to be easily manipulated. Indeed, the SoA presents a high number of research works related to several topics and a complex context. Besides, the reference regulatory framework comes as a maze of rules and prescriptions tough to follow and the current industrial applications are limited to certain conditions due to many different reasons. Therefore, the large amount of acquired information related to this topic needs to be managed and organized in a structured framework that enables the representation and exploitation of the knowledge.

\subsection{Knowledge management}

The knowledge management consists in the organization of the acquired knowledge into homogeneous "containers" and the definition of the relationship among the elements. Indeed, as depicted in the previous phase of the KBA, approaching to a collaborative workplace means to face a mass of information and a myriad of requirements all closely intertwined. Still, moving in this dense network of relationship and non-homogeneous information without a guide represents a real challenge. Therefore, the raw knowledge needs to be arranged and managed in order to make it structured, manageable and available.

This knowledge is arranged in a structured framework composed of two parts: the decomposition of the system and the construction of the relations.

The collaborative workplace is decomposed in a hierarchical form by means of a decomposition matrix. By the categorization of the information, the collected knowledge is organized in a framework in order to bring out both the main subjects of the topic and a well-structured skeleton of information. The resulting framework consists of five levels, summarized in Table 1. Therefore, all the knowledge results collected into a hierarchical matrix and structured in levels.

The knowledge associated to the collaborative workplace is divided in five main domains which collect all the homogeneous elements and respond to a specific need of the collaborative workplaces:

Table 1. Decomposition levels.

\begin{tabular}{|c|c|c|}
\hline Level & Name & Definition \\
\hline 1 & System & $\begin{array}{l}\text { The whole collaborative workplace, seen as a "sys- } \\
\text { tem" composed domains }\end{array}$ \\
\hline 2 & Domains & $\begin{array}{l}\text { Domains of the system that collect homogeneous } \\
\text { elements and respond to a specific need }\end{array}$ \\
\hline 3 & Elements & $\begin{array}{l}\text { Element of the sub-system not further decompos- } \\
\text { able and focused on specific aspects and face specific } \\
\text { functionalities }\end{array}$ \\
\hline 4 & Features & Characteristics and attribute of the elements \\
\hline 5 & Parameters & $\begin{array}{l}\text { Quantitative or qualitative values associated with } \\
\text { the features feature }\end{array}$ \\
\hline
\end{tabular}


- Logistic domain: refers to all issues related to the division and management of the workspaces, the inclusive and exclusive working areas, the available space and the management of the feeding.

- Technological domain: refers to all issues directly correlated to the active and passive resources, their features, number and characteristics.

- Safety and ergonomics domain: refers to the human wellness, safety working conditions and the performance of the control system, including the guards and protective devices.

- Process domain: refers to all issues related to the production, time, task sequencing, operations, interaction and communication between human and robot.

- Economic domain: refers to costs and benefits about the collaborative workplace, as well as the performances evaluation.

Belonging to these domains, twenty-one elements are presented below. They are parts or features of the collaborative workplace that face specific aspects of the problem and collect homogeneous information:

1. Physical limits (PL): available space and obstacles where the workplace is placed;

2. Workspaces (WS): layout of the workplace and division of the working areas;

3. Paths (P): accesses and exits of the workplace and paths;

4. Feeding (F): means of supplying material;

5. Workpiece (WP): main piece to be worked and components;

6. Equipment (EQ): furniture and instruments;

7. Usable devices (UD): control devices under operator control;

8. Operator $(\mathrm{O})$ : operator characteristics;

9. Robot (R): robot characteristics;

10. Autonomous guided vehicle (AGV): AGV characteristics;

11. Ergonomics (ER): ergonomic constraints and limitations;

12. Environment (EN): environmental working condition;

13. Minimum distances (MD): minimum distance set among both fixed and mobile resources;

14. Safeguarding perimeter (SP): physical or virtual workplace limit;

15. Safeguarding devices (SD): device not under operator control;

16. Type of work (TW): operation to carry out on the workpiece;

17. Task sequencing (TS): operations scheduled to be performed by human and robot;

18. Human-robot collaboration (HRC): interaction between human and robot;

19. Human machine interface (HMI): communication between human and robot;

20. Costs (C): economic constraints;

21. Benefits (B): key performance indicators (KPI).

Domains and elements represent the framework of the collaborative workplace (Table 2). Taking into account domains and elements, the designer can face all the aspects of the designing and he can address all the needed settings.

Once the structured framework is defined, the subsequent need is to establish a network of relationship among the elements. Therefore, the collaborative workplace is modelled as a graph $(\mathrm{G}=\mathrm{N}, \mathrm{E})$ [57] made of a set of nodes and edges [5]. Indeed, the adoption of the graph theory leads to highlight the connections among all the elements that are involved in the collaborative workplace. The relations among the nodes are highlighted by means of a direct graph (digraph) or an adjacency matrix (Table 3) where the values 1 or 0 means whether the elements on the row have a direct connection with the elements on the columns, or not $[58,59]$.

The first seven columns (workpiece, type of work, physical limits, ergonomics, environment, minimum distances and cost) are intended as source nodes, or external constraints. They are not influenced by the other nodes of the network. The central nodes are all configurations that depend on the designer choices. The last nodes are intended as outputs of the designing process, such as the workspaces and the path that 
Table 2. Decomposition Matrix.

\begin{tabular}{|c|c|c|c|c|c|}
\hline Domains & Needs & Groups & Macro aspects & Elements & Functionalities \\
\hline \multirow{4}{*}{ Logistic } & \multirow{4}{*}{ Management } & \multirow{2}{*}{ Layout } & \multirow{2}{*}{$\begin{array}{l}\text { Management of } \\
\text { spaces }\end{array}$} & Physical limits & Spatial constrain \\
\hline & & & & Workspaces & Space division \\
\hline & & \multirow{2}{*}{ Movement } & \multirow{2}{*}{$\begin{array}{l}\text { Management of } \\
\text { flow }\end{array}$} & Paths & Accessibility \\
\hline & & & & Feeding & Type of supplying \\
\hline \multirow{6}{*}{ Technologic } & \multirow{6}{*}{ Technic } & \multirow{3}{*}{$\begin{array}{l}\text { Passive } \\
\text { resources }\end{array}$} & \multirow{3}{*}{$\begin{array}{l}\text { Passive actor: } \\
\text { suffer the action }\end{array}$} & Workpiece & Target to achieve \\
\hline & & & & Equipment & Supportive object \\
\hline & & & & Usable devices & Operator support \\
\hline & & \multirow{3}{*}{$\begin{array}{l}\text { Active } \\
\text { resources }\end{array}$} & \multirow{3}{*}{$\begin{array}{l}\text { Active actor: act } \\
\text { the action }\end{array}$} & Operator & Operator features \\
\hline & & & & Robot & Robot features \\
\hline & & & & AGV & AGV features \\
\hline \multirow{5}{*}{$\begin{array}{l}\text { Safety \& } \\
\text { Ergonomics }\end{array}$} & \multirow{5}{*}{$\begin{array}{l}\text { Security \& } \\
\text { wellness }\end{array}$} & \multirow{2}{*}{ Human } & \multirow{2}{*}{ Human wellness } & Ergonomics & Operators constrain \\
\hline & & & & Environment & Working condition \\
\hline & & \multirow{3}{*}{ Safety } & \multirow{3}{*}{ Human security } & Minimum distances & Disposals constrain \\
\hline & & & & Safeguarding perimeter & Workplace border \\
\hline & & & & Safeguarding devices & Safety device \\
\hline \multirow{4}{*}{ Process } & \multirow{4}{*}{ Working } & \multirow{2}{*}{ Operation } & \multirow{2}{*}{$\begin{array}{l}\text { The aim of the } \\
\text { workplace }\end{array}$} & Type of work & Operation \\
\hline & & & & Task sequence & Relative operativity \\
\hline & & \multirow{2}{*}{ Interaction } & \multirow{2}{*}{$\begin{array}{l}\text { Human-robot } \\
\text { interaction }\end{array}$} & HRC & Type of interaction \\
\hline & & & & HMI & Communication \\
\hline \multirow{2}{*}{ Economic } & \multirow{2}{*}{ Economic } & \multirow{2}{*}{ Value } & \multirow{2}{*}{ Benefits vs. costs } & Costs & Economic constraint \\
\hline & & & & Benefits & Key Performance Indicators (KPI) \\
\hline
\end{tabular}

are the consequences of the previous decisions. Thus, a flow is generated that goes from the source nodes to the sink nodes. A user, following the flow inside this framework, can face all the aspects of the collaborative workplace designing.

\subsection{Knowledge representation}

The knowledge representation points to make the information collected and organized in the previous phases, understandable for the users in order to better implement actions and corrections. This phase embodies the selection of the software and methodologies to adopt and the logical implementation of the designing processes. The knowledge is manipulated by the user in order to focus on a certain part of the problem and represent a virtual prototype of the system. Consequently, the user can act on such a representation in order to extrapolate the needed information to refine the designing process and obtain a final consistent results. Furthermore, the application of the KBA can lead to the development of a designing paradigm as proposed in the following section.

The adoption of the graph theory allows easily manipulating the structured information and displaying it by means of tools like Matlab. The implementation of easy algorithms leads to the exploitation of the knowledge either visualizing on a sub-graph, that is a limited part of the whole graph, or focusing on a single node, by the study of its inputs and outputs. In the figure $3 a$, the relations among all the elements of a collaborative workplace (Table 3) are depicted as digraph. All the connections start from the "source nodes" that are considered external inputs or constraints for the designer.

Table 3. Adjacency matrix.

\begin{tabular}{|c|c|c|c|c|c|c|c|c|c|c|c|c|c|c|c|c|c|c|c|c|c|}
\hline & WP & TW & PL & ER & EN & MD & C & O & R & AGV & EQ & UD & TS & HRC & HMI & F & WS & P & SP & SD & B \\
\hline WP & 0 & 0 & 0 & 0 & 0 & 0 & 0 & 1 & 1 & 1 & 1 & 1 & 1 & 0 & 0 & 1 & 0 & 1 & 0 & 0 & 0 \\
\hline TW & 0 & 0 & 0 & 0 & 0 & 0 & 0 & 1 & 1 & 1 & 1 & 1 & 1 & 0 & 0 & 0 & 1 & 0 & 0 & 0 & 0 \\
\hline PL & 0 & 0 & 0 & 0 & 0 & 0 & 0 & 0 & 0 & 0 & 0 & 0 & 0 & 0 & 0 & 0 & 1 & 0 & 1 & 0 & 1 \\
\hline ER & 0 & 0 & 0 & 0 & 0 & 0 & 0 & 1 & 0 & 0 & 0 & 1 & 0 & 0 & 0 & 0 & 0 & 0 & 0 & 0 & 0 \\
\hline EN & 0 & 0 & 0 & 0 & 0 & 0 & 0 & 1 & 0 & 0 & 0 & 0 & 0 & 0 & 0 & 0 & 0 & 0 & 0 & 0 & 0 \\
\hline MD & 0 & 0 & 0 & 0 & 0 & 0 & 0 & 0 & 0 & 0 & 0 & 0 & 0 & 0 & 0 & 0 & 1 & 0 & 0 & 0 & 0 \\
\hline C & 0 & 0 & 0 & 0 & 0 & 0 & 0 & 1 & 1 & 1 & 1 & 1 & 0 & 0 & 0 & 0 & 0 & 0 & 0 & 1 & 1 \\
\hline O & 0 & 0 & 0 & 0 & 0 & 0 & 0 & 0 & 0 & 0 & 1 & 1 & 1 & 1 & 1 & 1 & 1 & 0 & 0 & 1 & 1 \\
\hline R & 0 & 0 & 0 & 0 & 0 & 0 & 0 & 0 & 0 & 0 & 1 & 1 & 1 & 1 & 1 & 0 & 1 & 0 & 0 & 1 & 1 \\
\hline AGV & 0 & 0 & 0 & 0 & 0 & 0 & 0 & 0 & 0 & 0 & 0 & 0 & 1 & 0 & 0 & 1 & 0 & 1 & 0 & 1 & 1 \\
\hline EQ & 0 & 0 & 0 & 0 & 0 & 0 & 0 & 0 & 0 & 0 & 0 & 0 & 0 & 0 & 0 & 0 & 1 & 0 & 0 & 0 & 1 \\
\hline UD & 0 & 0 & 0 & 0 & 0 & 0 & 0 & 0 & 0 & 0 & 0 & 0 & 0 & 0 & 0 & 0 & 0 & 0 & 0 & 0 & 1 \\
\hline TS & 0 & 0 & 0 & 0 & 0 & 0 & 0 & 0 & 0 & 0 & 0 & 0 & 0 & 0 & 0 & 0 & 1 & 0 & 0 & 0 & 1 \\
\hline HRC & 0 & 0 & 0 & 0 & 0 & 0 & 0 & 0 & 0 & 0 & 0 & 0 & 0 & 0 & 0 & 0 & 1 & 0 & 0 & 1 & 1 \\
\hline HMI & 0 & 0 & 0 & 0 & 0 & 0 & 0 & 0 & 0 & 0 & 0 & 1 & 0 & 0 & 0 & 0 & 0 & 0 & 0 & 0 & 1 \\
\hline F & 0 & 0 & 0 & 0 & 0 & 0 & 0 & 0 & 0 & 0 & 0 & 0 & 0 & 0 & 0 & 0 & 1 & 1 & 0 & 0 & 1 \\
\hline WS & 0 & 0 & 0 & 0 & 0 & 0 & 0 & 0 & 0 & 0 & 0 & 0 & 0 & 0 & 0 & 0 & 0 & 1 & 1 & 0 & 1 \\
\hline P & 0 & 0 & 0 & 0 & 0 & 0 & 0 & 0 & 0 & 0 & 0 & 0 & 0 & 0 & 0 & 0 & 0 & 0 & 1 & 1 & 1 \\
\hline SP & 0 & 0 & 0 & 0 & 0 & 0 & 0 & 0 & 0 & 0 & 0 & 0 & 0 & 0 & 0 & 0 & 0 & 0 & 0 & 0 & 0 \\
\hline SD & 0 & 0 & 0 & 0 & 0 & 0 & 0 & 0 & 0 & 0 & 0 & 0 & 0 & 0 & 0 & 0 & 0 & 0 & 0 & 0 & 0 \\
\hline B & 0 & 0 & 0 & 0 & 0 & 0 & 0 & 0 & 0 & 0 & 0 & 0 & 0 & 0 & 0 & 0 & 0 & 0 & 0 & 0 & 0 \\
\hline
\end{tabular}




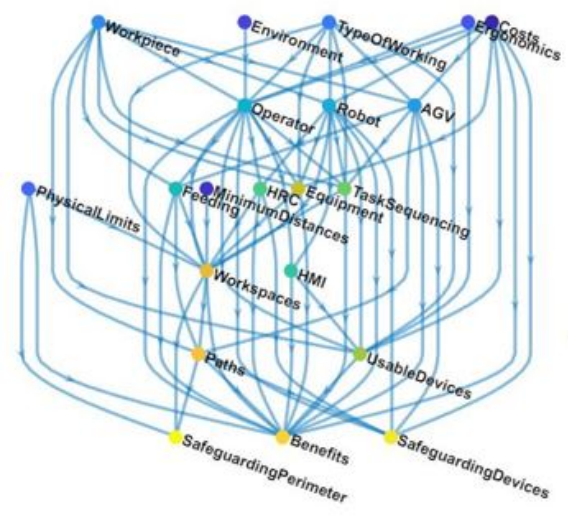

a

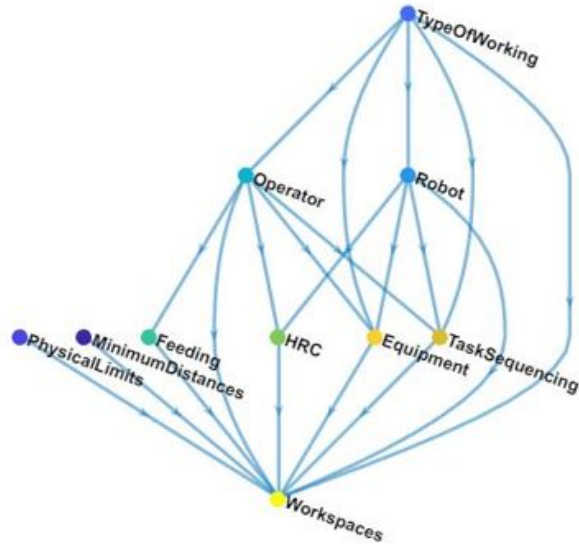

b

Figure 3. Relations among the elements of a collaborative workplace: a) complete network; b) input of node "workspaces".

The successive nodes need the information from the previous ones to be set. The figure $3 b$ is a focus on a specific node and presents all the inputs of the node "workspaces" that is about the layout and the working areas. These inputs are: Physical limits, Minimum distances, Type of working, Task sequencing, HRC, Robot, Operator, Equipment, and Feeding. They are the necessary information for the creation of the layout and the working areas.

Therefore, thanks to the decomposition of the problem and the use of the graph theory, the knowledge about collaborative workplace becomes available and manageable for those that are interested either in all the aspects of the problem or only a limited part.

The information and knowledge obtained from the KBA are used in the following to develop a modelling paradigm for the layout generation and optimization. The focus of the paradigm is the node workspaces that is about the working areas and division of the spaces. The output of this node is the layout of the collaborative workplace.

\section{The layout designing of the collaborative workplace}

In the context of Human-Robot Collaboration, accordingly to the topics presented in the previous section, the workplace layout planning is a strenuous challenge as well as a central theme for the design of collaborative workplaces. An efficient HRC layout must guarantee minimum movements, minimum travel time from one point to another, maximum utilization of resources and the safety of human beings. Several parameters, constraints, and issues must be taken into account to achieve efficient collaboration between human beings and robots. The KBA has provided a strong framework that results useful to identify these critical elements affecting the layout planning. Thus, a modelling paradigm is defined by exploiting the acquired and managed knowledge, and then, a systematic approach to the HRC layout design is developed according to the definitions and the concepts provided by the proposed paradigm.

\subsection{Modelling paradigm}

The paradigm results from the exploitation of the collected knowledge on the collaborative robotics field. The paradigm stems from the need to find a general model of collaborative workplace. Standard archetypes are proposed to provide design criteria and constraints, making up for the lack of a univocal reference scheme. By means of problem decomposition on a side, and knowledge management on the other, it is possible to focus on the main elements which affect the design of HRC workplaces. The modelling paradigm contains rigorous definitions of these elements, analyses their relationships and clarifies how they affect the layout design. The guiding principle 
of this process is to look at workplace layout as a set of entities and identify the main models describing how they must be linked to their actual counterparts.

The following subsections present the elements composing the paradigm, the relationships among them, and the influence they exert on the HRC layout planning.

\subsubsection{Elements of modelling paradigm}

The collaborative workplace is modelled defining (i) the physical entities that are part of it; (ii) the performed operations, on the one hand in terms of elementary tasks, and on the other, in terms of the type of robotic application; (iii) the workspaces.

More specifically, the collaborative workplace is described in terms of its tangible components [60]. It consists of resources - a stock or supply of materials, staff, and other assets - that can be drawn on in order to carry on tasks on certain workpieces. The resources may be active or passive resources [41]. Active resources are defined as resources which are trained to perform a certain task. Robot systems and humans are active resources. On another hand, the passive resources such as fixtures, static structures, tables, logistic resources and even machine tools, are used to perform a certain task. The workpiece is the object of the performed tasks. As already established by J. A. Marvel et al. [60], it can be composed of the principal component and any sub-components.

Then, the workplace is analysed from a process-related perspective. The operation carried out in the workplace is described in terms of elementary tasks. After, the task are classified by type, and by level of interaction between human being and robot.

Three main task types are defined:

- Process task is a valued-added task; it can be performed by humans and/or robots, using either elementary tools or even machine tools.

- Transport task is a material handling task; it can be performed by human, cobot, mobile manipulators, simple AGVs, as well as by means of passive resources such as conveyors.

- Control task does not contribute materially to the actual processing; it have to be entrusted to human being since it concerns cobots control tasks. It could be performed by means of a human machine interface (HMI) such as pendant controller, smartwatch, tablet or computer.

Tasks are further characterized by level of interaction between human and robot. Based on the classifications already given in [30], this work proposes four level of interaction:

- Independent tasks: human and robot perform different tasks on different workpieces.

- Sequential tasks: human and robot perform different tasks on the same workpiece placed in the same position. They share the same workspace but in different time (robot is inactive when human enters the collaborative space).

- Parallel tasks: human and robot perform separate tasks for the same goal at the same time. There is no physical contact between the human operator and the robot system. This level includes tasks which are performed inline.

- Collaborative tasks: human and robot work cooperatively in order to complete the processing of a single workpiece. Contact is allowed (but not strictly necessary) since robot and human can work "hand-in-hand".

Furthermore, The collaborative workplace is characterized according to the use made of the cobot(s) within it. The most common applications for which cobots installed by key vendors are employed, are showed in Figure 4. These applications are grouped in four main macro-categories according to their similarities, as follows: (i) Material handling when cobots are involved in the transport of material (e.g., pick and place, machine tending, packaging, palletizing, parts orientations); (ii) Assembly/Disassembly when cobots perform screwdriving, nut fastening, etc.; (iii) Precision Machining when cobots perform soldering, gluing, milling, cutting, etc.; (iv) Inspection when cobots 


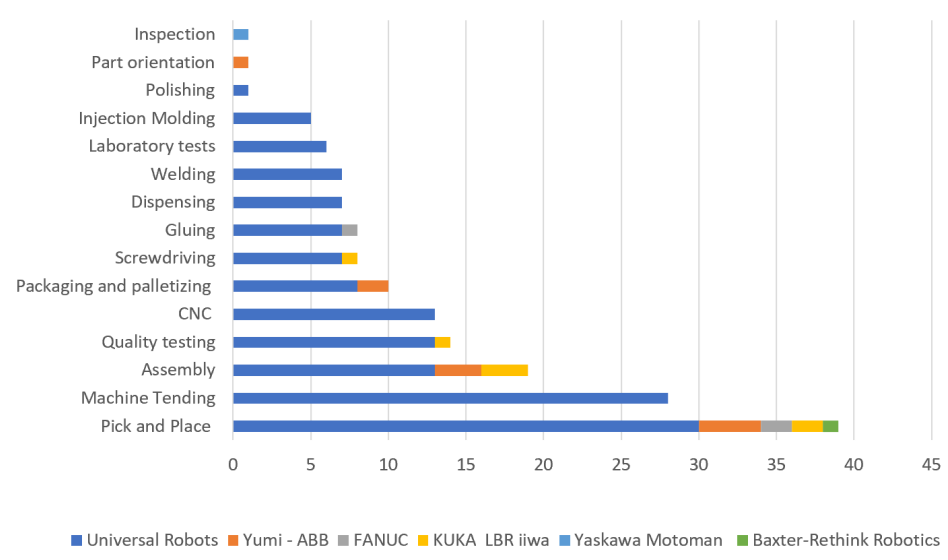

Figure 4. Most common applications for cobots installed by the key vendors

perform quality testing, non-destructive testing and so on. Obviously, a workplace could be composed of multiple cobots used for different categories of application.

Finally, the collaborative workplace is seen as the combination of several main workspaces: (i) the elementary workspaces dedicated to human operator and cobot, (ii) the composed workspaces identified through the combination of the elementary ones [5], and (iii) the logistic spaces.

Elementary workspaces. The collaborative workplace includes two elementary workspaces dedicated respectively to human and cobot.

Human Space (HS) is the space where the operator can perform his tasks; it is the result of the envelope of N Unitary Human Spaces (UHSs), each dedicated to a specific task. The UHS is a circular area around the operator with a diameter equal to the arm span (AS). The AS is defined as the measurement of the length from one end of a person's arms to the other, when raised parallel to the ground at shoulder height, at a $90^{\circ}$ angle. The space occupied by the operator is described by means of the body ellipse whose minor and major axes respectively represent depth and width of the human body. To be thorough, it is necessary to consider the increase in space due to the operator's clothing [61]. Zones of convenient reach (ZRC) are the areas that can be reached comfortably through movements that do not involve effort. These areas are included inside the UHSs and contain the Human Task Area (HTA). The HTA is a circular area dedicated to carry out the tasks which compose the whole operation. The center of this circular area is called Human Task Center (HTC). Figure 5 depicts the above-defined areas.

Robot Space (RS) is the space needed by the robot system (including attached tool and workpiece) to perform all its tasks. This space does not necessarily correspond to the maximum space that the robot can reach. It is usually defined through safety-rated soft axis and space limiting, as described in ISO 2018-1:2011. Practically, the robot system motion is software-defined and the space limiting is used to identify a space of any geometric shape within which robot motion is limited [25]. However, Robot space may be seen as the composition of $\mathrm{N}$ elementary robot spaces one for each task. The center of each intended space is named Robot Task Center (RTC).

Composed workspaces. Composed workspaces are obtained from the combination of the elementary workspaces: Collaborative Space (CS) is the intersection of the Human and Robot Space. It is present only if one of the established tasks is carried out in collaborative modes. In this case, it is possible to define a Collaborative Task Center (CTC) as the center of the CS.

Operational space (OS) is the sum of the elementary spaces and represents the space needed to perform all the intended tasks. It includes the Collaborative Space.

Logistic spaces. The spaces through which the workplace interfaces with the parts coming from the outside and the materials directed toward the outside are defined as logistic spaces. The logistic spaces are classified as follows: 


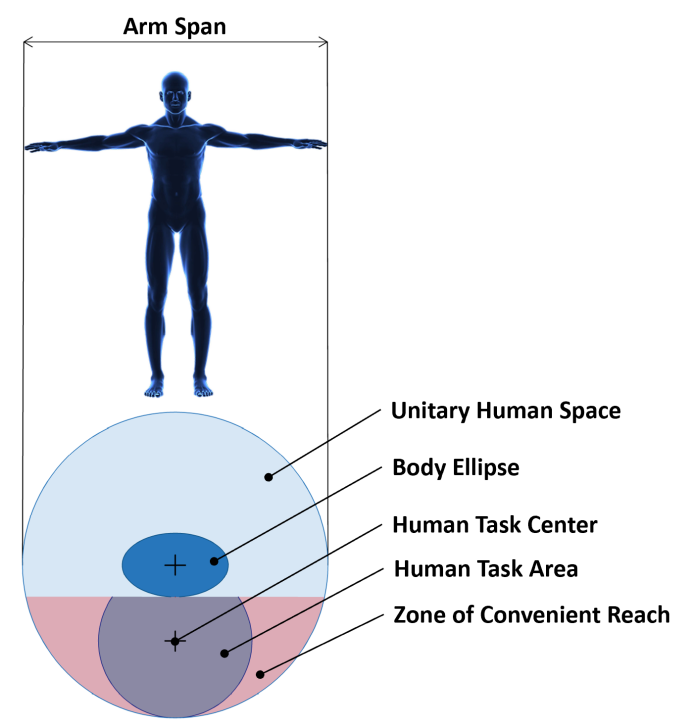

Figure 5. Human Space representation

1. The infeed spaces receive from the outside the workpiece, supply materials as screws, nuts, bolts, single parts to be assembled, sub-assemblies to be completed, groups to be processed.

2. The outfeed spaces receive the processed parts directed outwards. The outfeed spaces dedicated to correctly processed parts should be different from the outfeed spaces dedicated to parts which do not satisfy quality standards and must be reworked or discarded.

Shape and size of the logistic spaces are dependent on the geometric features of the logistic resources used to load/unload, to storage and to handle the workpieces. The position of the logistic spaces depends on the specific case. A valid solution could be to place the logistic spaces on the workplace sides. The possible scenarios are the following (Figure 6):

1. Infeed and outfeed spaces are placed near consecutive sides;

2. Infeed and outfeed spaces are placed near opposite sides;

3. Infeed and outfeed spaces are placed near the same side.

However, continuous flow manufacturing systems do not fall under the above classification. For example, in the automotive field, the assembly process usually involves the movement of the principal part on a central conveyor and the arrival of the sub-assemblies through suitable logistic resources. Therefore, on the one hand, the sub-assemblies arrive at workplace side in the infeed spaces, on the other hand, the principal part crosses the workplace following a defined path, entering from one specific point and leaving another.

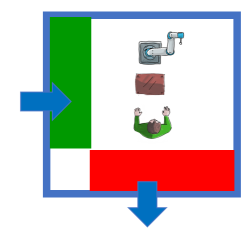

a

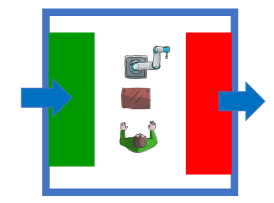

b

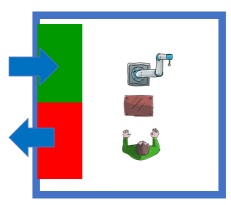

Outfeed space

Infeed space

$\downarrow$ Possible direction of material flow

Workplace perimeter

Figure 6. Possible position of Logistic Spaces: a) infeed and outfeed spaces are on consecutive sides; $b$ ) infeed and outfeed spaces are on opposite side; c) infeed and outfeed spaces are on the same side. 
Considering that both the robot and human can pick up parts from the logistic spaces to carry on the assigned tasks, it's clear that these spaces can be included entirely or be part of the elementary spaces.

Table 4 recapitulates synthetically the elements that are part of the proposed modelling paradigm.

\subsubsection{The relationships among the elements of the paradigm}

Every element of the paradigm affects the layout planning in a specif way; their influences on the whole workplace and the relationships among them are described below.

Each of the categories of application identified in the previous paragraph corresponds to a basic configuration of the workplace. Therefore, each basic configuration is characterized by some main elements and feature that are always present for that specific category. All the cobots used for a specific application are characterized by the same main features. Moreover, each category of application may prefer a specific level of interaction. For example, assembly applications mostly involve human being and cobot carrying out tasks in close contact. This means that tasks are mostly performed on a single item cooperatively and Human and Robot Spaces inevitably intersect.

The definition of the "type" and "level of interaction" for each task inherently provides information about the relative position between human and cobot within the workplace. Combining the information about the task attributes with the main configuration related to the category of application, a rough workplace layout begins to take place.

The characteristics of the resources, as well as those of the workpieces, influence the definition of the entire layout. The number, the size, the reach of the robot, affect the definition of the workspaces. The position within the workplace of the resources depends on some of their characteristics.

Table 4. Elements of modelling paradigm.

\begin{tabular}{|c|c|c|}
\hline \multicolumn{3}{|c|}{ Modeling paradigm elements } \\
\hline \multirow{3}{*}{ Workplace components } & Active resources & $\begin{array}{l}\text { Human operator } \\
\text { Cobot }\end{array}$ \\
\hline & Passive resources & $\begin{array}{l}\text { Fixtures } \\
\text { Machine tools }\end{array}$ \\
\hline & Workpieces & $\begin{array}{l}\text { Main part } \\
\text { Subcomponents }\end{array}$ \\
\hline \multirow[b]{2}{*}{ Task attributes } & Task type & $\begin{array}{l}\text { Process task } \\
\text { Transport task } \\
\text { Control task }\end{array}$ \\
\hline & Level of interaction & $\begin{array}{l}\text { Independent } \\
\text { Sequential } \\
\text { Parallel } \\
\text { Collaborative }\end{array}$ \\
\hline \multirow{4}{*}{ Category of application } & Material handling & e.g., pick and place, machine tending, palletizing \\
\hline & Assembly & e.g., nut fastening, screwdriving \\
\hline & Precision machining & e.g., welding, soldering, gluing, milling \\
\hline & Inspection & e.g., quality testing \\
\hline \multirow{3}{*}{ Workspaces } & Elementary workspaces & $\begin{array}{l}\text { Human Space } \\
\text { Robot Space }\end{array}$ \\
\hline & Composed workspaces & $\begin{array}{l}\text { Collaborative Space } \\
\text { Operational Space }\end{array}$ \\
\hline & Logistic Space & $\begin{array}{l}\text { Infeed Space } \\
\text { Outfeed Space }\end{array}$ \\
\hline
\end{tabular}


Workspaces are mostly influenced by the other elements of the paradigm. Position, size and shape of the workspaces depend on the characteristics of the tasks and workplace components. However, the logistic space features affect not only the layout but also the whole process; they ease or hinder it causing unreachability, too long distances to cover and so on.

In short, the paradigm can serve three kinds of purposes:

1. Describes the relevant aspects of the workplaces;

2. Simplifies the implementation of design methods.

3. Identifies changes in the layout of the workplaces;

\subsection{Problem formalization}

It is possible to formalize the HRC workplace layout design problem by introducing the following entities derived from the paradigm definitions:

1. Set of passive resources $P$, located within the workplace floor with pose defined as $\left\{x_{p_{i}}, y_{p_{i}}, \theta_{p_{i}}\right\} \forall p_{i} \in P$; each passive resource can be characterized by a set of points of interest, e.g., geometrical center of gravity, vertices and points reachable by active resources;

2. Set of active resources $A$, i.e., robots and human operators, each one characterized by a series of attributes and skills;

3. Set of elementary tasks that have to be performed by the active resources or by using passive resources (e.g. machine tools); each task is described by type and level of interaction;

4. Set of task centers within the workplace $W$ with pose expressed as $\left\{x_{w_{i}}, y_{w_{i}}\right\} \forall w_{i} \in$ W;

5. Set of basic locations within the workplace that correspond to the position where workpieces enter/exit the workplace.

Therefore, considering the above definitions, the solution to the design of the layout of the collaborative workplace could be expressed in matrix form by the following entities:

1. A matrix $T_{t c}$ of task-task center assignments;

2. A matrix $L_{c p}$ of task center-passive resource assignments;

3. A matrix $F_{a p}$ of active resource-passive resource assignments.

This means that the proposed method helps the designer in defining the optimised and standard compliant layout by identifying the task centers and defining their stances within the workplace according to an optimisation criterion.

The optimisation problem consists of finding a vector $w$ that minimizes a nonlinear objective function $f(w)$ subject to nonlinear constraints and bound constraints. It is known in the literature as a constrained nonlinear programming problem (NLP) [62]. The constraints are expressed by means of inequalities or equalities.

Therefore, it is possible to formulate the current problem as:

$$
\min _{w} f(w) \text { subject to }\left\{\begin{array}{l}
g(w) \leq 0 \\
g e q(w)=0 \\
l b \leq w \leq u b
\end{array}\right.
$$

with

$$
f(w)=a_{1} E(w)+a_{2} S(w)+a_{3} G(w) ;
$$

where $w$ is the vector containing the Cartesian coordinates of the identified task centers; $g(w)$ and $g e q(w)$ represent the constraints as equalities and inequalities; $l b$ and $u b$ stand for lower bounds and upper bounds. In the Equation 2, E(w) takes into account the ergonomic aspect, $S(w)$ is the occupied floor space within the workplace and $G(w)$ is an additional indicator; $a_{1}, a_{2}$ and $a_{3}$ are weighting factors. 


\subsection{Proposed Approach}

The main purpose of the current paper is to deal with the HRC workplace layout problem in a systematic and structured fashion, by means of the adoption of standardized reference models. The resulting approach is an iterative process, consisting of 4 main phases (Figure 7): (i) task analysis; (ii) task centers allocation; (iii) constrained optimization problem solving; (iv) rough layout definition. Downstream of the layout generation, a risk assessment could highlight the necessity of further risk-reduction measures, e.g. additional safety sensors, changes to the task sequencing, resources, and environment. Therefore, the layout designing is not a linear process since the iteration loops to previous phases are fundamental. The process is described in the following, where each phase is denoted at the beginning of the indention in italic.

Task analysis. Assuming that the task scheduling and resources allocation are defined in advance, the present methodology involves the analysis of the task scheduling as the first step of the whole designing process. The analysis of the task scheduling aims to identify the entities previously described: the process is described in terms of elementary tasks, each one characterized by "typology" and "level of interaction"; the complete set of resources and the corresponding tasks are identified; the reach, the payload, the size

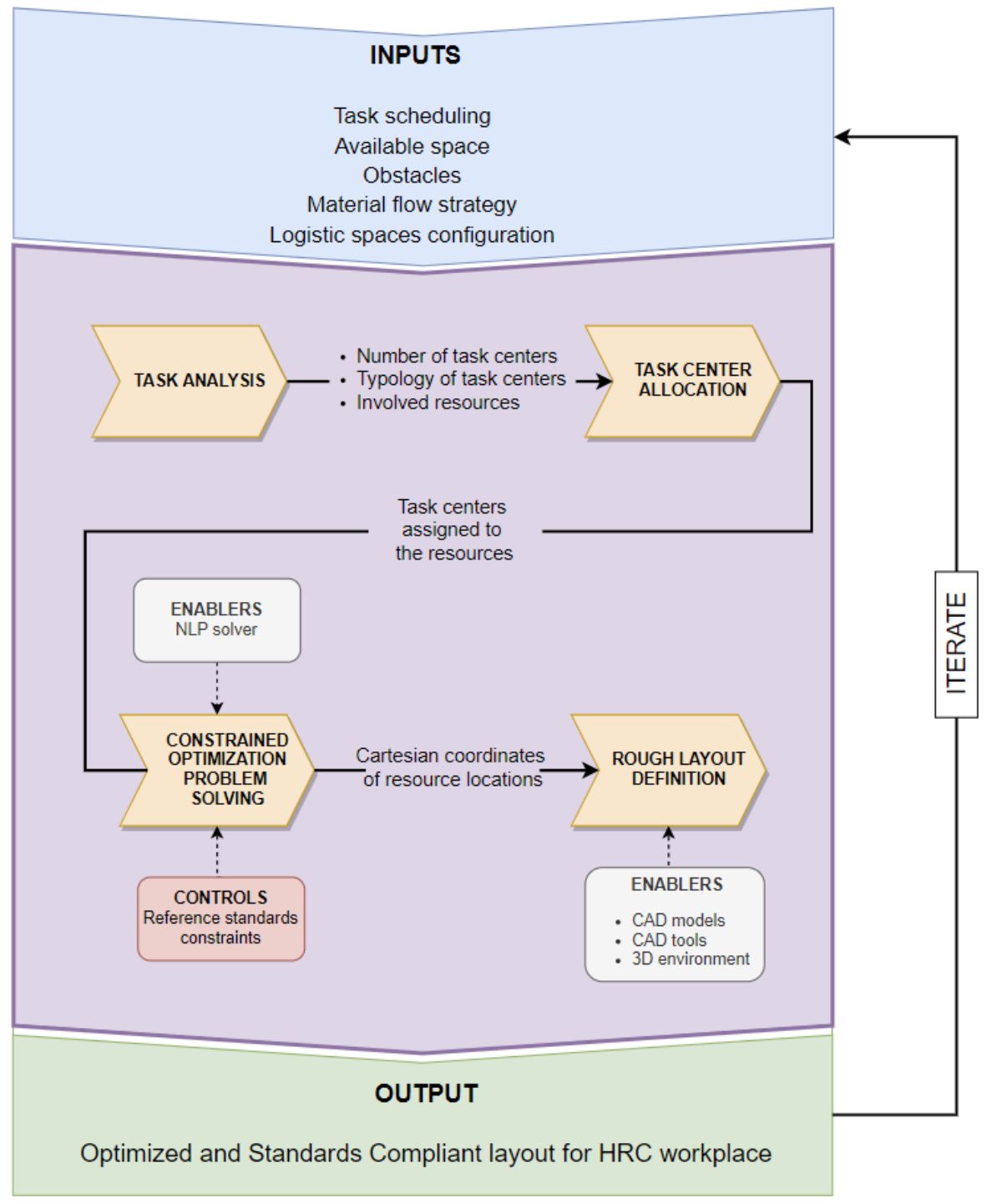

Figure 7. The proposed approach for the layout designing of HRC workplace. 
and the maximum speed of the robot, as well as the percentile, the gender of the human being and the dimensions of the passive resources are defined.

The final goal of this process is the definition of the matrix $T_{t c}$ containing the number and the type (i.e., HTC, RTC or CTC) of the task centers associated with the corresponding tasks. Every task is characterized by type and level of interaction between human operator and robot. The combination of this information returns the desired output. In particular, "transport" task implicates two task centers, one as the starting point and one as the arrival point, whether it is a robot task or a human task. "Process" and "control" tasks implicate one task center each. The level of interaction between human and robot establishes whether the task centers are coincident or not: for "independent" task, since human and robot perform different tasks on different workpieces, HTC and RTC are different; if the interaction between human and robot is at "sequential" level, HTC and RTC coincide in the space but in different time (robot and human are active one at time); the "parallel" level of interaction entails simultaneous tasks, aimed at the same goal, in different task centers; "collaborative" level of interaction means robot and human working in close contact and therefore coincident task centers.

Task center allocation. This phase entails the association between task centers and passive resources allowing to define the matrix $L_{c p}$. The task center defined for a specific operation is made to coincide with a point of interest belonging to the relative passive resource. Hence, this point becomes the origin of a local reference frame anchored to the resource. The local frame is then positioned and oriented with respect to the global reference. The authors have assumed the point of interest as the geometric center of gravity of the resource. Hence, the position of the passive resource required to perform a specific task can be determined by defining the position of the task center related to that specific task.

Constrained optimization problem solving. In the previous subsection the goal function to be minimized was defined by the Equation 2. That function is the sum of several terms related to specific features of the workplace. Currently, the present paper takes into account only the aspect related to the occupied floor space $S(w)$. The occupied floor space minimization criterion guarantees the best solution when it is necessary to reduce the dimension of the workplace and it also goes to meet the need to reduce the distances covered by the human operator in order to enhance the ergonomic conditions during the execution of his tasks. Ergonomic aspects will be considered in more detail in the future developments of this approach.

Therefore, considering the workplace floor space utilisation [41] as:

$$
F S=\left(x_{\max }-x_{\min }\right)+\left(y_{\max }-y_{\min }\right),
$$

where $x$ and $y$ are the maximum and the minimum coordinates reached by any resource in the plane, the goal function is defined as the sum of the Euclidean distances between the pairs of task centers:

$$
f=\sum_{k=1}^{b} \sqrt{\left(x_{k, i}-x_{k, j}\right)^{2}+\left(y_{k, i}-y_{k, j}\right)^{2}} .
$$

The $x_{i}, y_{i}$ and $x_{j}, y_{j}$ in the Equation 4 are the Cartesian coordinates of the $i$-th and $j$-th task centers respectively; $b$ represents the number of simple combinations given by:

$$
\left(\begin{array}{l}
n \\
2
\end{array}\right)=\frac{n !}{2 !(n-2) !}
$$

where $\mathrm{n}$ is the number of tasks centers to be placed in the available space.

Minimising the Euclidean distances between the task centers means that the resources are placed closer together, and consequently, the occupied floor space is also reduced. 
Obviously, the problem is constrained to place the resources within the available space and to avoid overlap of them. Furthermore, the solution of the optimisation problem is bound to compliance with the following provisions established by the reference standards:

- The minimum distance between moving objects, robot system and building areas, structures, users and other machines should be at least $500 \mathrm{~mm}[26,63]$.

- The minimum required separation distance between human and robot established by the ISO/TS15066 [27] with regard to speed and separation monitoring is:

$$
S_{p}(t)=S_{r}+S_{r}+S_{s}+C+Z_{d}+Z r
$$

The standard clarifies well how each term is determined.

- $\quad$ Safety distances are required to guarantee escape routes [64].

- The positioning and the orientation of the control device (e.g., the Human Machine Interface) should be such that the view on the robot is always unobstructed (as declared in the Annex A of ISO 10218-2 [26] and ISO 13850 [65]).

- Maximum load carrying distance should be defined depending on the carried cumulative mass [66].

Moreover, logistic space, which contains the logistic resources, can be bound to assume one of the three configuration described in the section 4.1.

In short, the mathematical modelling for the optimization problem is set up as the search for the minimum value of a nonlinear function of $n \times 2$ variables subject to nonlinear constraints. The constraints are expressed by means of equalities and inequalities. The $n \times 2$ variables correspond to the Cartesian coordinates $(x, y)$ of the plan projection of the $\mathrm{n}$ task centers associated with the relative passive resources. The problem is solved by means of a Matlab nonlinear programming solver.

Rough layout definition. This phase outcome is the optimised and standard-compliant principal solution to the HRC layout designing problem. Once the optimization problem is solved, and the passive resources are placed, the working areas are determined by defining the matrix $F_{a p}$ that associates the active resources to the employed passive resources. The proposed approach identifies as the main workspaces the Human Space and the Robot Space, and using the definitions proposed in the paradigm, it is easy to mark them. Conceptually, the first attempt layout could look like the solution shown in Figure 8. As it can be seen from the figure, the task centers (i.e., HTC, RTC, CTC) and the related passive resources, whose poses are determined by solving the NLP problem, the Human Space, the Robot Space, and the Logistic Space, where the workpieces enter and exit the workplace, are explicitly identified.

\section{Case study}

In this paragraph, the approach presented above is used for designing a HRC workplace layout for quality inspection of welding points on automotive components. Then, a what-if analysis is presented to evaluate how the layout is affected by changing some input parameters.

\subsection{Collaborative workplace for inspection of welded parts}

The proposed case study concerns the welding points quality check carried out by means of ultrasonic technology. It involves the adoption of a human operator and a cobot. The operator is concerned with taking and carrying the component whereas the cobot executes the inspection. The cobot is equipped with a specially designed ultrasonic end-effector. The workpiece to be inspected requires a precise adjustment on the dedicated stand. After the inspection, the operator picks up the component from the dedicated stand and deposits it on the outfeed stand. The main activities of the operator are: carrying the component, precise positioning it and monitoring as long as the cobot execute the inspection. 


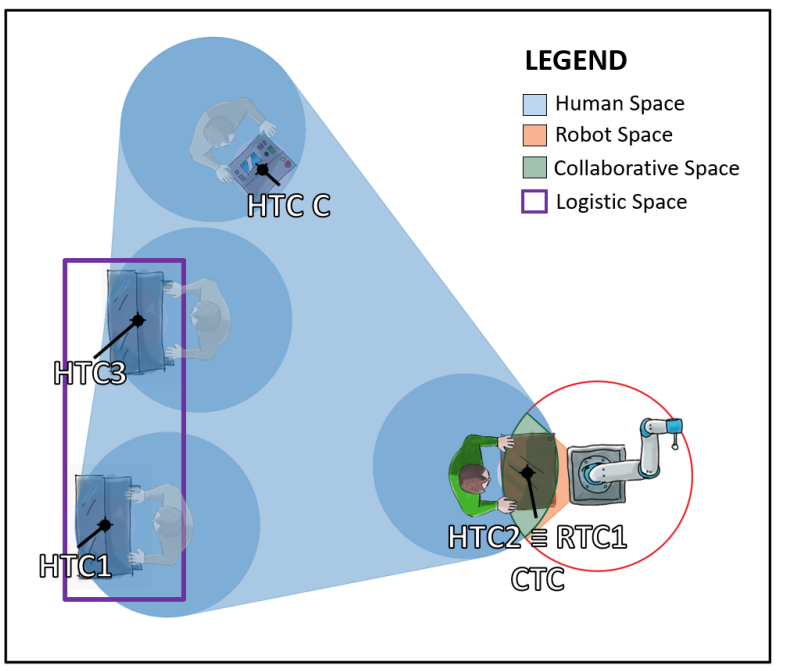

Figure 8. Conceptual layout of the HRC workplace

The four steps of the proposed approach, i.e., (i) task analysis, (ii) task centers allocation, (iii) constrained optimisation problem solving, (iv) rough layout definition, are applied aiming at designing a standard compliant and optimized layout for this case study.

Task analysis. The task scheduling (Figure 9) is analysed as the main input of the entire process. The task scheduling analysis allows defining:

1. Tasks of which the entire operation is composed;

2. Resources assigned to each task;

3. Type and number of active resources;

4. Type and number of passive resources;

5. Information about workpieces;

6. Robot characteristics;

7. Tasks attributes (type, level of interaction, temporal order);

8. Number and type of task centers according to the procedures described in the previous chapter.

Table 5 indicates the information obtained from the task analysis including the task centers list related to all tasks.

The chosen collaborative robot is a UR10 cobot produced by Universal Robot, with performance level "d", category 3 according to ISO 13849 [67]. It has 6 rotational joints, a payload of $10 \mathrm{~kg}$ and 1.3 meters of maximum reach. The cobot is equipped with an end-effector tool which enables the robot to accomplish the inspection activity. A

\begin{tabular}{|c|c|c|c|}
\hline ID & Task & \begin{tabular}{|l|l} 
Resource \\
\end{tabular} & Time \\
\hline 1 & Positioning the workpiece on the inspection stand & 3 & \\
\hline 2 & Checking the correct postitioning & 3 & \\
\hline 3 & selecting the inspection plans & 3 & \\
\hline 4 & Entering of the workpiece ID number & 3 & \\
\hline 5 & Starting the control cycle & 3 & \\
\hline 6 & Actieving all inspection postitions & $\underbrace{9}$ & \\
\hline 7 & Monitoring the operation & 3 & \\
\hline 8 & Laading the workpiece on the outreed stand & 3 & \\
\hline
\end{tabular}

Figure 9. Task scheduling related to the quality check of welding points performed in a collaborative manner. 
Table 5. Analysis of the task scheduling.

\begin{tabular}{|c|c|c|c|c|c|}
\hline ID & Task & Task Type & $\begin{array}{c}\text { Active } \\
\text { Resource }\end{array}$ & $\begin{array}{c}\text { Level of } \\
\text { Interaction }\end{array}$ & Task center \\
\hline 1 & $\begin{array}{l}\text { Positioning the } \\
\text { workpiece on } \\
\text { the inspection stand }\end{array}$ & Transport & $\begin{array}{l}\text { Human } \\
\text { Operator }\end{array}$ & Sequential & HTC 1-HTC 2 \\
\hline 2 & $\begin{array}{l}\text { Checking the correct } \\
\text { positioning }\end{array}$ & Process & $\begin{array}{l}\text { Human } \\
\text { Operator }\end{array}$ & Sequential & HTC 2 \\
\hline 3 & $\begin{array}{l}\text { Selecting the } \\
\text { inspection plans }\end{array}$ & Control & $\begin{array}{l}\text { Human } \\
\text { Operator }\end{array}$ & Sequential & HTC C \\
\hline 4 & $\begin{array}{l}\text { Entering workpiece } \\
\text { ID number }\end{array}$ & Control & $\begin{array}{l}\text { Human } \\
\text { Operator }\end{array}$ & Sequential & HTC C \\
\hline 5 & $\begin{array}{l}\text { Starting the } \\
\text { control cycle }\end{array}$ & Control & $\begin{array}{l}\text { Human } \\
\text { Operator }\end{array}$ & Sequential & HTC C \\
\hline 6 & $\begin{array}{l}\text { Achieving } \\
\text { all inspection } \\
\text { positions }\end{array}$ & Process & $\begin{array}{l}\text { Cobot } \\
\text { UR10 }\end{array}$ & Collaborative & RTC 1 = HTC2 \\
\hline 7 & $\begin{array}{l}\text { Monitoring } \\
\text { the operation }\end{array}$ & Process & $\begin{array}{l}\text { Human } \\
\text { Operator }\end{array}$ & Collaborative & HTC 2 \\
\hline 8 & $\begin{array}{l}\text { Loading the } \\
\text { workpiece on } \\
\text { the outfeed stand }\end{array}$ & Transport & $\begin{array}{l}\text { Human } \\
\text { Operator }\end{array}$ & Sequential & HTC 2 - HTC 3 \\
\hline
\end{tabular}

dedicated Human Machine Interface (HMI) simplifies the cobot control and improves the Human-Robot Collaboration.

The set of required passive resources is also defined in this first phase. The following passive resources are needed:

1. Infeed stand;

2. Outfeed stand;

3. Inspection stand;

4. Robot pedestal;

5. Human Machine Interface.

According to the proposed method, "transport" task needs two task centers: the first task, i.e., "loading workpiece in the control station", needs the Human Task Center 1 where the operator takes the workpiece, and the Human Task Center 2 where the operator places it. The following tasks are "process" or "control" tasks, performed sequentially on the same workpiece. Therefore, they are all executed at Human Task Center 2 and Human Task Center $C$. Task 6 is assigned to the cobot which has to achieve all the inspection positions. The cobot carries out the task under the human supervision, in a collaborative manner. This means that the Robot Task Center 1 coincides with the Human Task Center 2, identifying a Collaborative Task Center. Besides, task 7, linked to Human Task Center 2, is the collaborative task performed by the human being. It does not involve physical contact between the resources, but it is carried on in the same workspace, on the same workpiece and at the same time. However, the minimum separation distance is guaranteed. Finally, tasks 3, 4 and 5 are "control" tasks. They don't contribute to the actual process but concern the cobot control. The related task center is different from those previously identified. It affects the position of the HMI within the available space. Task 8, i.e., "loading workpiece in the storage station", is treated as task 1 since it involves the transport of the workpiece from Human Task Center 2 to Human Task Center 3.

Task center allocation. Then, the passive resource, which is needed to carry out a specific task, is associate with the task center related to that task. In particular, the infeed and outfeed stands are associated respectively to the Human Task Center 1 and 3, whereas the inspection stand is associated to Human Task Center 2 (that coincides with Robot Task 
Center 1). Finally, the Human-Machine Interface is associated to Human Task Center C. The robot pedestal supports the cobot.

Constrained optimization problem solving. This phase consists in defining the task centers position. As a result, the related passive resources are also placed. The available space is a rectangular area of 49 square meters. There are no pre-existing obstacles. The position of the logistic spaces is defined considering the adjacent workplaces. The solution of the problem of constrained optimisation, which minimizes the occupied floor space, is found by means of a Matlab solver.

Rough layout definition. The elementary workspaces are defined in the last step. The envelope of the four Unitary Human Spaces (UHSs), which are needed to carry out all the tasks assigned to human, identifies the Human Space. The Robot Space is softwaredefined. The intersection between the elementary spaces identifies the Collaborative Space, while their union identifies the Operational Space. The logistic spaces intersect the Human Space. Infeed space and outfeed space are placed on the same side according to the adjacent workplaces position. Figure 10 shows the layout obtained by applying the proposed method. At the end of the design process, the workplace can be completed with additional fixtures and safety devices if these are really needed.

\subsection{What-if analysis}

In this section, a what if analysis that involves the generation and subsequent evaluation of several scenarios is presented. In the first stage, the proposed approach is used to generate several collaborative workplaces starting from different sets of inputs. In particular, three input parameters are fixed on three levels in order to create 27 different combinations. Consequently a critical assessment of them is carried out through some criteria in order to find out the best configuration and combination of parameters. Afterwards, the impact of the selected parameters on the performances of the system is evaluated. For this purpose, four Key Performance Indicators (KPIs) of the workplace are evaluated by means of specific functions. Then, an utility value given by the combination of that functions is obtained by means of the Simple MultiAttribute Rating Technique (SMART) [68-70]. This technique is a synthetic technique that

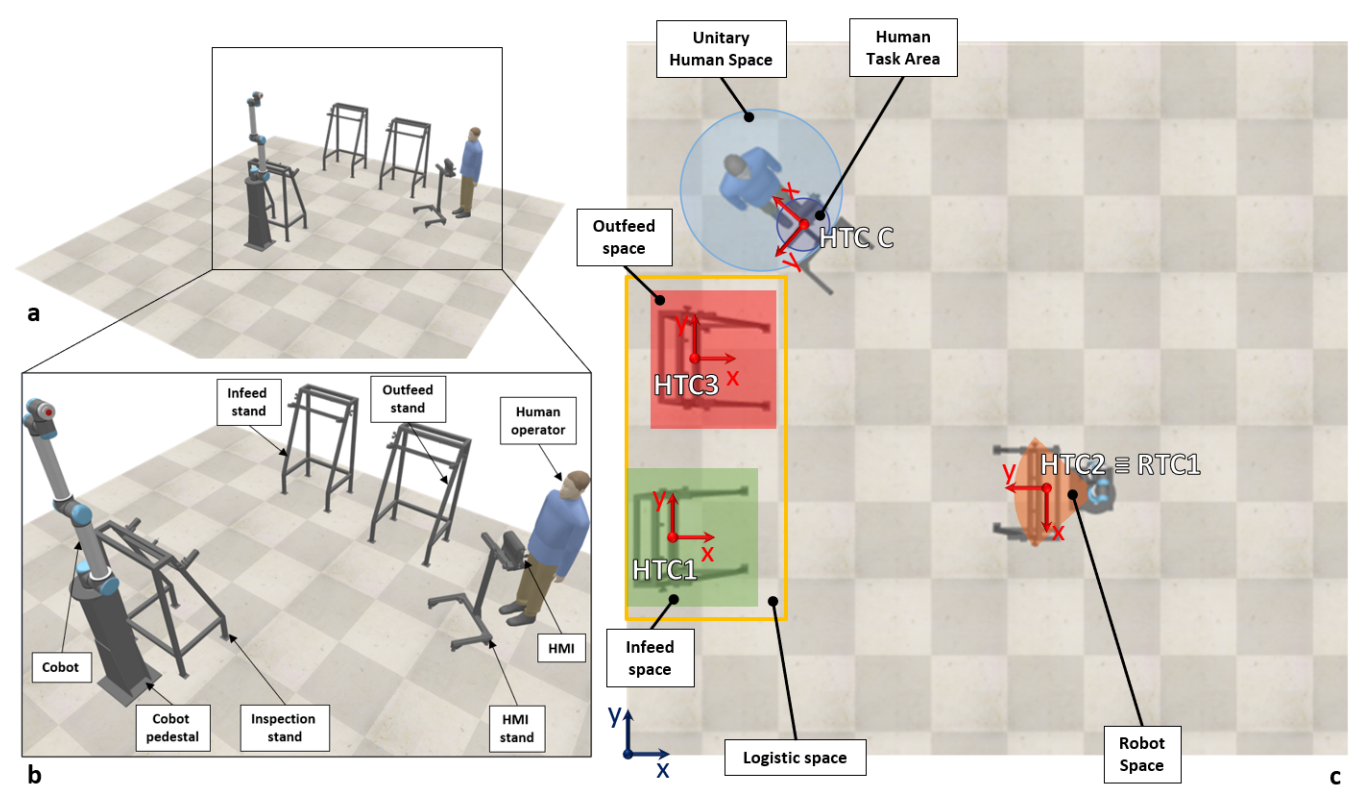

Figure 10. The HRC workplace layout obtained by means of the proposed approach: a) the workplace showed in the 3D environment; b) 3D enlargement showing the several resources that are part of the workplace; c) $2 \mathrm{D}$ view highlighting the several task centers and the main workspaces. 
combines several non-homogeneous quantities and provides an utility value through four steps:

- $\quad$ assigning a weight to each function or performance by means of a comparison among them;

- making the output value of each function dimensionless and on a normalized scale making the best of each function corresponded to the maximum in the normalized scale;

- $\quad$ multiplying the dimensionless values and the weights;

- $\quad$ summing the results obtained in the previous step.

The output value of this technique is a weighted combination of the other performances and the highest value identifies the best configuration among to the different combinations of parameters.

The input parameters of this analysis, selected as control factors, are:

1. Minimum distance: the minimum distance among the resources,

2. Robot speed: the speed adopted by the robot to carry out the inspection

3. Logistic areas: the relative position of the logistic areas.

According to the ISO 13854 [63], the minimum distance among the resources must not be lower than $500 \mathrm{~mm}$. In the context of the spatial optimization, it represents the lower limit. On another hand, according to the designing and production purposes, it could be required this value higher to guarantee specific needs. Indeed, even tough the optimization algorithm points to the minimization of the distances, there is always a minimum value to be guaranteed, not only due to the safety, but also for the working exigences. Then, the robot speed influences several key aspects of the workplace such as the timing, the safety, the proximity of the human to the robot and the collaborative time. Finally, the relative position of the logistic areas, as explained in section 4.1 .1 can be set at the same side, consecutive side or opposite side in a single workplace, with an impact on the logistic optimization that depends on the manufacturing context.

The selected parameters belong to a specific domain of the collaborative workplace (Table 2). The minimum distances is a safety requirement, the robot speed is a technical parameter related to the active resource, and the logistic areas is a logistic parameter. All these parameters, or factors, are presented in Table 6 with the selected values and description.

By the combination of these factors, a Design of Experiment (DoE) is made with a total of 27 combinations. Such configurations need to be evaluated through different performances with a set of functions. Consequently, an utility value is calculated for each configuration with the SMART method in order to provide a representative score. The proposed functions are: (i) the impact on space, (ii) the HRC relevance, (iii) the time cycle and (iv) the initial investment cost. Three of that functions need to be minimized whereas the HRC relevance to be maximized. Such functions represent performances with competitive priorities, that means the improvement of one implies the worsening of another. They are reported in Table 7 with their expressions and the descriptions.

The impact on space compares the real occupied space with a reference value. In this study the reference value is $49 \mathrm{~m}^{2}$. The time is the sum of the single time of all the

Table 6. Inputs and levels for Design of Experiments analysis.

\begin{tabular}{|c|c|c|c|c|c|}
\hline ID & Inputs & Level 1 & Level 2 & Level 2 & Summary \\
\hline 1 & $\begin{array}{l}\text { Minimum } \\
\text { distance }\end{array}$ & $500 \mathrm{~mm}$ & $700 \mathrm{~mm}$ & $900 \mathrm{~mm}$ & $\begin{array}{l}\text { Minimum distances between } \\
\text { two generic resources }\end{array}$ \\
\hline 2 & $\begin{array}{l}\text { Robot } \\
\text { speed }\end{array}$ & $\begin{array}{l}0.25 \\
\mathrm{~m} / \mathrm{s}\end{array}$ & $0.5 \mathrm{~m} / \mathrm{s}$ & $0.75 \mathrm{~m} / \mathrm{s}$ & $\begin{array}{l}\text { Speed adopted by the robot } \\
\text { to carry out the inspection }\end{array}$ \\
\hline 3 & $\begin{array}{l}\text { Logistic } \\
\text { areas. }\end{array}$ & $\begin{array}{l}\text { Same } \\
\text { side }\end{array}$ & $\begin{array}{l}\text { Consecutive } \\
\text { side }\end{array}$ & $\begin{array}{l}\text { Opposite } \\
\text { side }\end{array}$ & $\begin{array}{l}\text { Relative position of the infeed } \\
\text { and outfeed areas }\end{array}$ \\
\hline
\end{tabular}


Table 7. Evaluation functions [37,41].

\begin{tabular}{|c|c|c|c|}
\hline Name & Function & Objective & Summary \\
\hline $\begin{array}{l}\text { Impact } \\
\text { on space }\end{array}$ & $\frac{\left(x_{\max }-x_{\min }\right)\left(y_{\max }-y_{\min }\right)}{A_{t}} 100$ & Minimize & $\begin{array}{l}\text { Percentage of the total plant } \\
\text { available area occupied by the } \\
\text { collaborative workplace }\end{array}$ \\
\hline $\begin{array}{l}\text { HRC rele- } \\
\text { vance }\end{array}$ & $\frac{T_{c}}{T_{t}} 100$ & Maximize & $\begin{array}{l}\text { Percentage of the total needed ex- } \\
\text { ecution time characterized by the } \\
\text { simultaneous working of human } \\
\text { and robot }\end{array}$ \\
\hline Time & $\sum_{i=1}^{N} t_{i}$ & Minimize & Total needed execution time \\
\hline Cost & $\sum_{i=1}^{R} c_{i} n_{i}$ & Minimize & $\begin{array}{l}\text { Total cost as the sum of all the } \\
\text { active and passive resources }\end{array}$ \\
\hline
\end{tabular}

sequential tasks. The HRC relevance is provided by the percentage of the collaborative time on the total time. Finally, the cost is the sum of the costs of each resource on the workplace.

All the 27 configurations generated by means of the proposed method are firstly evaluated by the four functions. Consequently, the SMART is adopted to obtain a synthetic score. The necessary weights assigned to the functions, in accordance to the relative importance of each function on the collaborative workplace performances, are the following:

- Impact on space: $38 \%$

- HRC relevance: $13 \%$

- Time: $13 \%$

- Cost: $38 \%$

Indeed, the industrial experience reached by means of a discussion with a selected group of experts of the sector, suggests to pose a higher attention on the minimization of spaces and costs.

\section{Results and Discussion}

By the combination of the three parameters presented in the previous section, 27 scenarios are generated through the method presented in the section 4 . Figure 11 presents three example of the generated layout. In particular, the different position of the logistic areas is present on the three configuration.
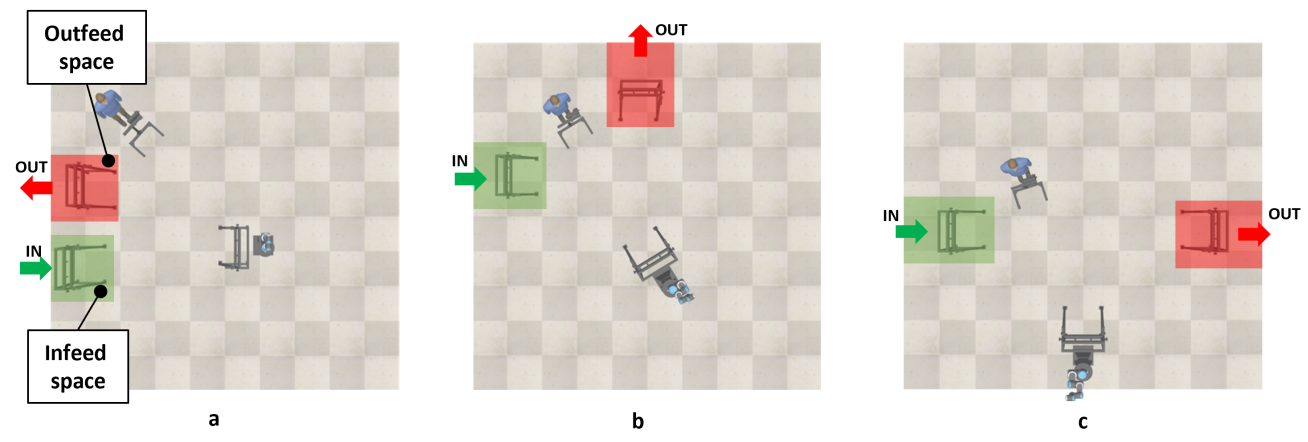

Figure 11. Three different workplace configurations obtained by changing the position of the logistic spaces: a) infeed and outfeed spaces are placed on the same side; b) infeed and outfeed spaces are placed on consecutive sides; c) infeed and outfeed spaces are placed on opposite sides. 
Table 8. Design of Experiment and results.

\begin{tabular}{|c|c|c|c|c|}
\hline Conf. & Minimum distance & Robot speed & Logistic areas & Result \\
\hline 1 & $500 \mathrm{~mm}$ & $0.25 \mathrm{~m} / \mathrm{s}$ & Same side & 87.16 \\
\hline 2 & $500 \mathrm{~mm}$ & $0.25 \mathrm{~m} / \mathrm{s}$ & Consecutive side & 84.39 \\
\hline 3 & $500 \mathrm{~mm}$ & $0.25 \mathrm{~m} / \mathrm{s}$ & Opposite side & 59.77 \\
\hline 4 & $500 \mathrm{~mm}$ & $0.5 \mathrm{~m} / \mathrm{s}$ & Same side & 88.54 \\
\hline 5 & $500 \mathrm{~mm}$ & $0.5 \mathrm{~m} / \mathrm{s}$ & Consecutive side & 85.63 \\
\hline 6 & $500 \mathrm{~mm}$ & $0.5 \mathrm{~m} / \mathrm{s}$ & Opposite side & 60.11 \\
\hline 7 & $500 \mathrm{~mm}$ & $0.75 \mathrm{~m} / \mathrm{s}$ & Same side & 84.41 \\
\hline 8 & $500 \mathrm{~mm}$ & $0.75 \mathrm{~m} / \mathrm{s}$ & Consecutive side & 81.23 \\
\hline 9 & $500 \mathrm{~mm}$ & $0.75 \mathrm{~m} / \mathrm{s}$ & Opposite side & 55.18 \\
\hline 10 & $700 \mathrm{~mm}$ & $0.25 \mathrm{~m} / \mathrm{s}$ & Same side & 81.87 \\
\hline 11 & $700 \mathrm{~mm}$ & $0.25 \mathrm{~m} / \mathrm{s}$ & Consecutive side & 79.35 \\
\hline 12 & $700 \mathrm{~mm}$ & $0.25 \mathrm{~m} / \mathrm{s}$ & Opposite side & 58.45 \\
\hline 13 & $700 \mathrm{~mm}$ & $0.5 \mathrm{~m} / \mathrm{s}$ & Same side & 83.05 \\
\hline 14 & $700 \mathrm{~mm}$ & $0.5 \mathrm{~m} / \mathrm{s}$ & Consecutive side & 80.36 \\
\hline 15 & $700 \mathrm{~mm}$ & $0.5 \mathrm{~m} / \mathrm{s}$ & Opposite side & 58.83 \\
\hline 16 & $700 \mathrm{~mm}$ & $0.75 \mathrm{~m} / \mathrm{s}$ & Same side & 78.87 \\
\hline 17 & $700 \mathrm{~mm}$ & $0.75 \mathrm{~m} / \mathrm{s}$ & Consecutive side & 75.77 \\
\hline 18 & $700 \mathrm{~mm}$ & $0.75 \mathrm{~m} / \mathrm{s}$ & Opposite side & 53.93 \\
\hline 19 & $900 \mathrm{~mm}$ & $0.25 \mathrm{~m} / \mathrm{s}$ & Same side & 76.88 \\
\hline 20 & $900 \mathrm{~mm}$ & $0.25 \mathrm{~m} / \mathrm{s}$ & Consecutive side & 73.76 \\
\hline 21 & $900 \mathrm{~mm}$ & $0.25 \mathrm{~m} / \mathrm{s}$ & Opposite side & 57.39 \\
\hline 22 & $900 \mathrm{~mm}$ & $0.5 \mathrm{~m} / \mathrm{s}$ & Same side & 77.86 \\
\hline 23 & $900 \mathrm{~mm}$ & $0.5 \mathrm{~m} / \mathrm{s}$ & Consecutive side & 74.50 \\
\hline 24 & $900 \mathrm{~mm}$ & $0.5 \mathrm{~m} / \mathrm{s}$ & Opposite side & 57.80 \\
\hline 25 & $900 \mathrm{~mm}$ & $0.75 \mathrm{~m} / \mathrm{s}$ & Same side & 73.58 \\
\hline 26 & $900 \mathrm{~mm}$ & $0.75 \mathrm{~m} / \mathrm{s}$ & Consecutive side & 69.90 \\
\hline 27 & $900 \mathrm{~mm}$ & $0.75 \mathrm{~m} / \mathrm{s}$ & Opposite side & 58.94 \\
\hline
\end{tabular}

All the 27 scenarios are evaluated independently through the four KPIs presented in the section 5.2. The impact on space, the HRC relevance, the time and the cost are calculated for each configuration and a synthetic value given by the combination of them is pulled out by means of the SMART technique. In Table 8 are presented the results of this assessment. For brevity, only the combinations of parameters and the SMART utility value are reported.

According to the SMART, it emerged that the best configuration is the no. 4, characterized by:

- $\quad$ Minimum distance: $500 \mathrm{~mm}$;

- Robot speed: $0.5 \mathrm{~m} / \mathrm{s}$;

- Logistic areas: the same side.

On another hand, other configurations are considered the best according to the other KPIs. The configuration no. 1 is the one that has the highest score for impact on space and the HRC relevance, whereas the configuration no. 7 is the one that minimize the time. Furthermore, the cost is not considered because it provides the same value for all the configurations.

Therefore, looking at the best configurations to select, a preliminary assessment leads to the following consideration:

1. The minimum distance to consider among the resources should be as less as possible. Indeed a minimum distance of $500 \mathrm{~mm}$ is the best according all the evaluation functions and the utility value.

2. The relative position of the logistic areas are located at the same side for all the winning configurations. 
Table 9. Results of the ANOVA analysis.

\begin{tabular}{lrlr}
\hline \multicolumn{1}{c}{ ANOVA } & Analysis & \\
\hline \multicolumn{1}{c}{ SMART } & & \multicolumn{2}{c}{ Impact on space } \\
Minimum distance & $8.14 \%$ & Minimum distance & $8.93 \%$ \\
Robot speed & $2.98 \%$ & Robot speed & $0.40 \%$ \\
Logistic areas & $88.88 \%$ & Logistic areas & $90.68 \%$ \\
\hline \multicolumn{1}{c}{ HRC relevance } & & & Time \\
\hline Minimum distance & $0.00 \%$ & Minimum distance & $0.03 \%$ \\
Robot speed & $99.96 \%$ & Robot speed & $98.60 \%$ \\
Logistic areas & $0.04 \%$ & Logistic areas & $1.37 \%$ \\
\hline
\end{tabular}

3. The robot speed presents a different result. Indeed, the minimum value is considered the best according to the impact on space and the HRC relevance, the middle value is the best solution according to SMART and the maximum value is the best for the time.

Therefore, apparently, the best designing should follow the minimization of the distance among the resources and the positioning of the logistic areas at the same side. About the robot speed, it should be set according to the interest of the designer and the company. Nevertheless, an ANOVA analysis conducted on the results leads to discover the magnitude of the impact that the three parameters have on the functions, in order to find out which of them is the most influential. The analysis is carried out on the utility value and three on the four KPIs. The initial investment function is not considered because its value does not change for all the configurations. The principal main effect of the parameters as well as the combined effects are considered. The resume of the analysis is presented in Table 9. The final considerations are the following:

- The relative position of the logistic areas is very significant on the impact on space and the SMART utility value $(88,88 \%$ and $90,68 \%)$;

- The robot speed is very significant on the collaborative time and total time of execution $(99,96 \%$ and $98,60 \%)$;

- The minimum distance has a very low impact on all the performances (less than $9 \%$ on all the functions);

- No interaction effect is significant for all the performance (less that $1 \%$ ).

Therefore, from this analysis it emerges that the minimum distance is not a significant factor for all the evaluation criteria. Indeed it has a very low impact on all the evaluation functions and it could be not considered as input for the generation of the scenarios. Its value can depend only on the economic and feasibility needs.

On another hand, the robot speed is one of the factors that impacts mostly on the total time and the collaborative time, as expected. Indeed, if the robot moves at a lower speed, there are two different aspects that come out: (i) it is allowed to human to stay close to the robot area and (ii) the shared time could increase or decrease its percentage on the total time depending on the task scheduling. In contrast, if the robot move at the higher speed, it is forbidden to stay close to it, therefore the collaboration is penalized, even though the total cycle time decreases. Furthermore, if the robot task is carried out at the highest speed during the inspection, the control by the human is the worse possible.

Finally, the relative position of the logistic area is the most influential factor for SMART and impact on space. That is reasonable because the different layouts need different distribution of the resources on the space. Moreover, the impact on the space is the function with the highest weight in the SMART together with the cost. Therefore, the factor with the highest impact on such performances, has a comparable impact even on the SMART utility value. 


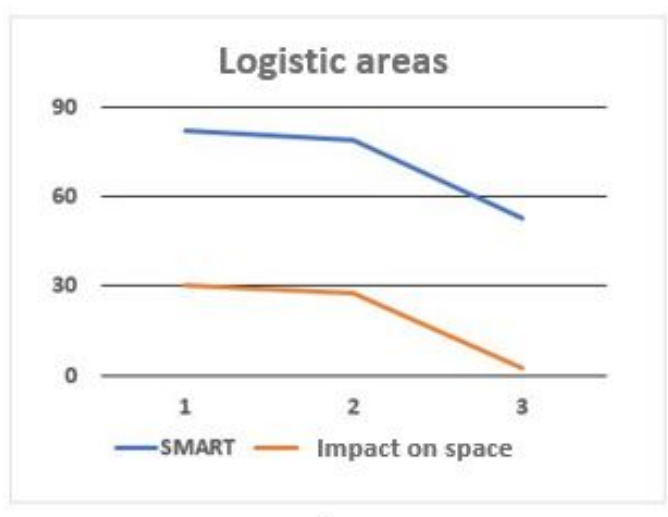

a

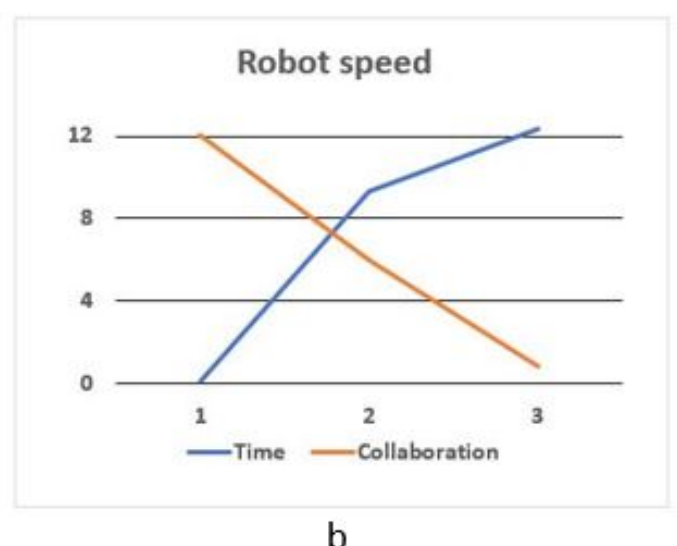

b

Figure 12. Mean principal effects: a) trend of the impact on space and SMART utility value according to the relative position of the logistic areas; b) trend of the total time and collaborative time according to the robot speed

In the figure 12, it is possible to see the trend of the functions according to their main influential factors. In figure 12a the trends of the SMART utility value and the impact on the space are reported according to the relative position of the logistic areas. They have pretty similar trends as explained before. In particular, it is possible to see that both the performances present very low changes when the logistic areas are located at the same side or consecutive sides, but they get worse when the logistic areas are located at opposite sides. This results suggests to adopt indifferently mainly logistic areas at the same side or consecutive sides. On another hand, to locate the logistic areas at opposite sides could lead to a worsening of the performance.

The matter is different for the robot speed. In figure $12 \mathrm{~b}$, the trend of total time and collaborative time are reported according to the robot speed. It is interesting to see that the collaborative time has a linear decreasing trend with the robot speed whereas the performance of the total time has a increasing trend.

The analysis on the results leads to the following considerations. The minimum distance among the resource is not a important control factor on the KPIs, therefore it can be set following the criteria of minimum cost or another criterion. The relative position of the logistic areas has a high impact on the use of space and the SMART utility value. In particular, when the logistic areas are at opposite sides, there is a general worsening of these performances. Still, the robot speed presents a conflicting result. It is impossible to maximize the performance of the total time and collaborative time at the same time, even though the middle robot speed is a good compromise to achieve the best possible condition for both the KPIs.

\section{Conclusions}

The paper presents a streamlined approach aimed at designing of collaborative workplace layout. Firstly, the context and the challenges of collaborative robotics are presented in order to define the boundaries of the problem. Consequently, the whole knowledge about collaborative robotics is systematically collected and managed, according to a KBA. A modelling paradigm is the outcome of this process; it allows the definition of a method for layout designing which leverages the positioning of the HTC and RTC in the available space, according to a defined optimization criterion. Finally, a what-if analysis is carried out by means of DoE, in order to generate a set of scenarios and evaluate them through four functions and one utility value.

The main contribution of the paper is the presentation of a modelling paradigm used to: (i) describe the relevant aspect common to several workplaces; (ii) simplify the implementation of design methods; (iii) identify changes in the layout of the workplaces. Hence, the designing approach, the aim of which is the spatial optimization and the 
standards compliance of the collaborative workplace layout, is proposed. This is applied to a collaborative workplace for quality ultrasonic inspection to prove its usefulness and feasibility. The case study allows showing the systematic usage of the designing approach, and carrying on a what-if analysis by generating multiple layout configurations. Three inputs are set as control factors: (i) the minimum distance among the resources, (ii) the robot speed and (iii) the relative position of the logistic areas. They are set on three levels. A Design of Experiment (DoE) made of 27 combinations is carried out and four Key Performance Indicators (KPIs) are selected to assess the layout alternatives. The KPIs simulated are: the impact on space, the execution time, the relevance of the collaboration (HRC relevance) and the initial investment cost. To obtain a utility value representative of each configuration, the SMART technique was adopted. Finally, an ANOVA analysis is carried out in order to find out the real impact of the control factors on each KPI. The results shows that there is one configuration that is eligible as the best configuration according the investigation, and only two control factors on three are very significant for the designing of the HRC workplace layout.

Author Contributions: All authors contributed equally to this work.

Funding: This study was developed with the economic support of MUR (Ministry of Universities and Research) under the remit of project ARS01_00861, "Integrated collaborative systems for smart factory - ICOSAF".

Conflicts of Interest: The authors declare no conflict of interest. The funders had no role in the design of the study; in the collection, analyses, or interpretation of data; in the writing of the manuscript, or in the decision to publish the results.

\section{References}

1. Nahavandi, S. Industry 5.0-A human-centric solution. Sustainability 2019, 11, 4371.

2. Parente, M.; Figueira, G.; Amorim, P.; Marques, A. Production Scheduling in the Context of Industry 4.0: Review and Trends. International Journal of Production Research 2020, 58, 5401-5431. doi:10.1080/00207543.2020.1718794.

3. Djuric, A.M.; Urbanic, R.; Rickli, J. A Framework for Collaborative Robot (CoBot) Integration in Advanced Manufacturing Systems. SAE International Journal of Materials and Manufacturing 2016, 9, 457-464. doi:10.4271/2016-01-0337.

4. Tan, Q.; Tong, Y.; Wu, S.; Li, D. Anthropocentric Approach for Smart Assembly: Integration and Collaboration. Journal of Robotics 2019, 2019, 1-8. doi:10.1155/2019/3146782.

5. Di Marino, C.; Rega, A.; Vitolo, F.; Patalano, S.; Lanzotti, A. A New Approach to the Anthropocentric Design of Human-Robot Collaborative Environments. ACTA IMEKO 2020, 9, 80-87. doi:10.21014/acta_imeko.v9i4.743.

6. Krüger, J.; Wang, L.; Verl, A.; Bauernhansl, T.; Carpanzano, E.; Makris, S.; Fleischer, J.; Reinhart, G.; Franke, J.; Pellegrinelli, S. Innovative Control of Assembly Systems and Lines. CIRP Annals 2017, 66, 707-730. doi:10.1016/j.cirp.2017.05.010.

7. Gualtieri, L.; Rauch, E.; Rojas, R.; Vidoni, R.; Matt, D.T. Application of Axiomatic Design for the Design of a Safe Collaborative Human-Robot Assembly Workplace. MATEC Web of Conferences 2018, 223, 01003. doi:10.1051/matecconf/201822301003.

8. Kusiak, A.; Heragu, S.S. The Facility Layout Problem. European Journal of Operational Research 1987, 29, 229-251. doi:10.1016/03772217(87)90238-4.

9. Drira, A.; Pierreval, H.; Hajri-Gabouj, S. Facility Layout Problems: A Survey. Annual Reviews in Control 2007, 31, 255-267. doi:10.1016/j.arcontrol.2007.04.001.

10. Shiller, Z. Optimal Robot Motion Planning and Work-Cell Layout Design. Robotica 1997, 15, 31-40. doi:10.1017/S0263574797000052.

11. Scimmi, L.S.; Melchiorre, M.; Troise, M.; Mauro, S.; Pastorelli, S. A Practical and Effective Layout for a Safe Human-Robot Collaborative Assembly Task. Applied Sciences 2021, 11, 1763.

12. Murali, P.; Darvish, K.; Mastrogiovanni, F. Deployment and Evaluation of a Flexible Human-Robot Collaboration Model Based on AND/OR Graphs in a Manufacturing Environment. Intelligent Service Robotics 2020, 13, 439-457. doi:10.1007/s11370-020-00332-9.

13. Mateus, J.; Claeys, D.; Limère, V.; Cottyn, J.; Aghezzaf, E.H. Base Part Centered Assembly Task Precedence Generation. International Journal of Advanced Manufacturing Technology 2020, 107, 607-616. doi:10.1007/s00170-019-04864-y.

14. Bänziger, T.; Kunz, A.; Wegener, K. Optimizing Human-Robot Task Allocation Using a Simulation Tool Based on Standardized Work Descriptions. Journal of Intelligent Manufacturing 2020, 31, 1635-1648. doi:10.1007/s10845-018-1411-1.

15. Makrini, I.E.; Merckaert, K.; Winter, J.D.; Lefeber, D.; Vanderborght, B. Task Allocation for Improved Ergonomics in Human-Robot Collaborative Assembly. Interaction Studies. Social Behaviour and Communication in Biological and Artificial Systems 2019, 20, 102-133. doi:10.1075/is.18018.mak.

16. Kim, W.; Peternel, L.; Lorenzini, M.; Babič, J.; Ajoudani, A. A Human-Robot Collaboration Framework for Improving Ergonomics During Dexterous Operation of Power Tools. Robotics and Computer-Integrated Manufacturing $2021,68$. doi:10.1016/j.rcim.2020.102084. 
17. Abobakr, A.; Nahavandi, D.; Hossny, M.; Iskander, J.; Attia, M.; Nahavandi, S.; Smets, M. RGB-D Ergonomic Assessment System of Adopted Working Postures. Applied Ergonomics 2019, 80, 75-88. doi:10.1016/j.apergo.2019.05.004.

18. Paletta, L.; Pszeida, M.; Nauschnegg, B.; Haspl, T.; Marton, R. Stress Measurement in Multi-Tasking Decision Processes Using Executive Functions Analysis. Advances in Intelligent Systems and Computing 2020, 953, 344-356. doi:10.1007/978-3-030-20473-0_33.

19. Colim, A.; Faria, C.; Braga, A.C.; Sousa, N.; Rocha, L.; Carneiro, P.; Costa, N.; Arezes, P. Towards an Ergonomic Assessment Framework for Industrial Assembly Workstations-A Case Study. Applied Sciences 2020, 10, 3048. doi:10.3390/app10093048.

20. Waters, T.; Putz-Anderson, V.; Garg, A.; Fine, L. Revised NIOSH Equation for the Design and Evaluation of Manual Lifting Tasks. Ergonomics 1993, 36, 749-776. doi:10.1080/00140139308967940.

21. Lynn, M.; Corlett, N. RULA: A Survey Method for the Investigation of Work-Related Upper Limb Disorders. Applied ergonomics 1993, 24, 91-99.

22. Occhipinti, E. OCRA: A Concise Index for the Assessment of Exposure to Repetitive Movements of the Upper Limbs. Ergonomics 1998, 41, 1290-1311. doi:10.1080/001401398186315.

23. Rojas, R.; Wehrle, E.; Vidoni, R. A Multicriteria Motion Planning Approach for Combining Smoothness and Speed in Collaborative Assembly Systems. Applied Sciences (Switzerland) 2020, 10. doi:10.3390/app10155086.

24. Matsas, E.; Vosniakos, G.C. Design of a Virtual Reality Training System for Human-Robot Collaboration in Manufacturing Tasks. International Journal on Interactive Design and Manufacturing (IJIDeM) 2017, 11, 139-153. doi:10.1007/s12008-015-0259-2.

25. ISO 10218-1:2011. Robots and Robotic Devices -Safety Requirements for Industrial Robots - Part 1: Robots. International Organization for Standardization: Geneva, Switzerland.

26. ISO 10218-2:2011. Robots and Robotic Devices - Safety Requirements for Industrial Robots - Part 2: Robot Systems and Integration. International Organization for Standardization: Geneva, Switzerland.

27. ISO \TS 15066:2016. Robots and Robotic Devices: Collaborative Robots. International Organization for Standardization: Geneva, Switzerland.

28. Bdiwi, M.; Pfeifer, M.; Sterzing, A. A New Strategy for Ensuring Human Safety during Various Levels of Interaction with Industrial Robots. CIRP Annals 2017, 66, 453-456. doi:10.1016/j.cirp.2017.04.009.

29. Malik, A.A.; Bilberg, A. Developing a Reference Model for Human-Robot Interaction. International Journal on Interactive Design and Manufacturing (IJIDeM) 2019, 13, 1541-1547. doi:10.1007/s12008-019-00591-6.

30. Aaltonen, I.; Salmi, T.; Marstio, I. Refining Levels of Collaboration to Support the Design and Evaluation of Human-Robot Interaction in the Manufacturing Industry. Procedia CIRP 2018, 72, 93-98. doi:10.1016/j.procir.2018.03.214.

31. El Zaatari, S.; Marei, M.; Li, W.; Usman, Z. Cobot Programming for Collaborative Industrial Tasks: An Overview. Robotics and Autonomous Systems 2019, 116, 162-180. doi:10.1016/j.robot.2019.03.003.

32. Matheson, E.; Minto, R.; Zampieri, E.G.G.; Faccio, M.; Rosati, G. Human-Robot Collaboration in Manufacturing Applications: A Review. Robotics 2019, 8, 100. doi:10.3390/robotics8040100.

33. Malik, A.; Brem, A. Digital Twins for Collaborative Robots: A Case Study in Human-Robot Interaction. Robotics and ComputerIntegrated Manufacturing 2021, 68. doi:10.1016/j.rcim.2020.102092.

34. Kousi, N.; Gkournelos, C.; Aivaliotis, S.; Lotsaris, K.; Bavelos, A.C.; Baris, P.; Michalos, G.; Makris, S. Digital Twin for Designing and Reconfiguring Human-Robot Collaborative Assembly Lines. Applied Sciences 2021, 11, 4620.

35. Wright, L.; Davidson, S. How to Tell the Difference between a Model and a Digital Twin. Advanced Modeling and Simulation in Engineering Sciences 2020, 7, 13. doi:10.1186/s40323-020-00147-4.

36. Boschetti, G.; Bottin, M.; Faccio, M.; Minto, R. Multi-Robot Multi-Operator Collaborative Assembly Systems: A Performance Evaluation Model. Journal of Intelligent Manufacturing 2021. doi:10.1007/s10845-020-01714-7.

37. Faccio, M.; Minto, R.; Rosati, G.; Bottin, M. The Influence of the Product Characteristics on Human-Robot Collaboration: A Model for the Performance of Collaborative Robotic Assembly. The International Journal of Advanced Manufacturing Technology 2020, 106, 2317-2331. doi:10.1007/s00170-019-04670-6.

38. Vitolo, F.; Pasquariello, A.; Patalano, S.; Gerbino, S. A Multi-Layer Approach for the Identification and Evaluation of Collaborative Robotic Workplaces Within Industrial Production Plants. Design Tools and Methods in Industrial Engineering; Springer International Publishing: Cham, 2020; Lecture Notes in Mechanical Engineering, pp. 719-730. doi:10.1007/978-3-030-31154-4_61.

39. Gualtieri, L.; Rauch, E.; Vidoni, R.; Matt, D.T. An Evaluation Methodology for the Conversion of Manual Assembly Systems into Human-Robot Collaborative Workcells. Procedia Manufacturing 2019, 38, 358-366. doi:10.1016/j.promfg.2020.01.046.

40. Gualtieri, L.; Palomba, I.; Merati, F.A.; Rauch, E.; Vidoni, R. Design of Human-Centered Collaborative Assembly Workstations for the Improvement of Operators' Physical Ergonomics and Production Efficiency: A Case Study. Sustainability 2020, 12, 3606. doi:10.3390/su12093606.

41. Tsarouchi, P.; Michalos, G.; Makris, S.; Athanasatos, T.; Dimoulas, K.; Chryssolouris, G. On a Human-Robot Workplace Design and Task Allocation System. International Journal of Computer Integrated Manufacturing 2017, 30, 1272-1279. doi:10.1080/0951192X.2017.1307524.

42. Ore, F.; Sánchez, J.L.J.; Wiktorsson, M.; Hanson, L. Design Method of Human-Industrial Robot Collaborative Workstation with Industrial Application. International Journal of Computer Integrated Manufacturing 2020, 33, 911-924. doi:10.1080/0951192X.2020.1815844.

43. Lietaert, P.; Billen, N.; Burggraeve, S. Model-Based Multi-Attribute Collaborative Production Cell Layout Optimization. 2019 20th International Conference on Research and Education in Mechatronics (REM); IEEE: Wels, Austria, 2019; pp. 1-7. doi:10.1109/REM.2019.8744136. 
44. Mateus, J.C.; Claeys, D.; Limère, V.; Cottyn, J.; Aghezzaf, E.H. A Structured Methodology for the Design of a HumanRobot Collaborative Assembly Workplace. The International Journal of Advanced Manufacturing Technology 2019, 102, $2663-2681$. doi:10.1007/s00170-019-03356-3.

45. Saenz, J.; Behrens, R.; Schulenburg, E.; Petersen, H.; Gibaru, O.; Neto, P.; Elkmann, N. Methods for Considering Safety in Design of Robotics Applications Featuring Human-Robot Collaboration. International Journal of Advanced Manufacturing Technology 2020, 107, 2313-2331. doi:10.1007/s00170-020-05076-5.

46. Gervasi, R.; Mastrogiacomo, L.; Franceschini, F. A Conceptual Framework to Evaluate Human-Robot Collaboration. International Journal of Advanced Manufacturing Technology 2020, 108, 841-865. doi:10.1007/s00170-020-05363-1.

47. Ralyté, J.; Jeusfeld, M.; Backlund, P.; Kühn, H.; Arni-Bloch, N. A Knowledge-Based Approach to Manage Information Systems Interoperability. Information Systems 2008, 33, 754-784. doi:10.1016/j.is.2008.01.008.

48. Favi, C.; Garziera, R.; Campi, F. A Rule-Based System to Promote Design for Manufacturing and Assembly in the Development of Welded Structure: Method and Tool Proposition. Applied Sciences 2021, 11, 2326. doi:10.3390/app11052326.

49. Li, Z.; Liang, P.; Avgeriou, P. Application of Knowledge-Based Approaches in Software Architecture: A Systematic Mapping Study. Information and Software Technology 2013, 55, 777-794. doi:10.1016/j.infsof.2012.11.005.

50. Aulinas, M.; Nieves, J.; Cortés, U.; Poch, M. Supporting Decision Making in Urban Wastewater Systems Using a Knowledge-Based Approach. Environmental Modelling and Software 2011, 26, 562-572. doi:10.1016/j.envsoft.2010.11.009.

51. Ansari, F.; Hold, P.; Khobreh, M. A Knowledge-Based Approach for Representing Jobholder Profile toward Optimal Human-Machine Collaboration in Cyber Physical Production Systems. CIRP Journal of Manufacturing Science and Technology 2020, 28, 87-106. doi:10.1016/j.cirpj.2019.11.005.

52. Michalos, G.; Makris, S.; Tsarouchi, P.; Guasch, T.; Kontovrakis, D.; Chryssolouris, G. Design Considerations for Safe HumanRobot Collaborative Workplaces. Procedia CIRP 2015, 37, 248-253. doi:10.1016/j.procir.2015.08.014.

53. Villani, V.; Pini, F.; Leali, F.; Secchi, C. Survey on Human-Robot Collaboration in Industrial Settings: Safety, Intuitive Interfaces and Applications. Mechatronics 2018, 55, 248-266. doi:10.1016/j.mechatronics.2018.02.009.

54. Matthias, B.; Reisinger, T. Example Application of ISO/TS 15066 to a Collaborative Assembly Scenario. Proceedings of ISR 2016: 47st International Symposium on Robotics, 2016, pp. 1-5.

55. Bauer, W.; Bender, M.; Braun, M.; Rally, P.; Scholtz, O. Lightweight Robots in Manual Assembly-Best to Start Simply. Examining Companies' Initial Experiences with Lightweight Robots. Frauenhofer-Institut für Arbeitswirtschaft und Organisation IAO, Stuttgart 2016, 63 .

56. Itd, R.a.M. Global Collaborative Robots Market 2020-2024 - Research and Markets.

57. Deo, N. Graph Theory with Applications to Engineering and Computer Science; Dover Publications : Made available through hoopla: United States, 2017.

58. Majeed, A.; Rauf, I. Graph Theory: A Comprehensive Survey about Graph Theory Applications in Computer Science and Social Networks. Inventions 2020, 5, 10. doi:10.3390/inventions5010010.

59. Schaeffer, S.E. Graph Clustering. Computer Science Review 2007, 1, 27-64. doi:10.1016/j.cosrev.2007.05.001.

60. Marvel, J.A.; Falco, J.; Marstio, I. Characterizing Task-Based Human-Robot Collaboration Safety in Manufacturing. IEEE Transactions on Systems, Man, and Cybernetics: Systems 2015, 45, 260-275. doi:10.1109/TSMC.2014.2337275.

61. Pheasant, S. Human, Anthropometry, Ergonomic and Design, Translated by: Choobineh A, Moedi M. Tehran, Markaz Publication 1997.

62. Bazaraa, M.; Sherali, H.; Shetty, C. Nonlinear Programming: Theory and Algorithms; Nonlinear Programming: Theory and Algorithms, 2005; p. 853. doi:10.1002/0471787779.

63. ISO 13854:2017. Safety of Machinery - Minimum Gaps to Avoid Crushing of Parts of the Human Body. International Organization for Standardization: Geneva, Switzerland.

64. ISO 12100:2010. Safety of Machinery - General Principles for Design - Risk Assessment and Risk Reduction. International Organization for Standardization: Geneva, Switzerland.

65. ISO 13850:2015. Safety of Machinery - Emergency Stop Function - Principles for Design. International Organization for Standardization: Geneva, Switzerland.

66. ISO 11228-1:2003. Ergonomics - Manual Handling - Part 1: Lifting and Carrying. International Organization for Standardization: Geneva, Switzerland.

67. ISO 13849-1:2015. Safety of machinery - Safety-related parts of control systems - Part 1: General principles for design. International Organization for Standardization: Geneva, Switzerland.

68. Siregar, D.; Arisandi, D.; Usman, A.; Irwan, D.; Rahim, R. Research of Simple Multi-Attribute Rating Technique for Decision Support. Journal of Physics: Conference Series 2017, 930, 012015. doi:10.1088/1742-6596/930/1/012015.

69. Risawandi.; Rahim, R. Study of the Simple Multi-Attribute Rating Technique For Decision Support. International Journal of Scientific Research in Science and Technology 2016, 2, 491-494.

70. Barron, F.H.; Barrett, B.E. The Efficacy of SMARTER — Simple Multi-Attribute Rating Technique Extended to Ranking. Acta Psychologica 1996, 93, 23-36. doi:10.1016/0001-6918(96)00010-8. 